\title{
Mobilité et subsistance au Magdalénien dans le Bassin de l'Aude
} Laure Fontana

\section{Citer ce document / Cite this document :}

Fontana Laure. Mobilité et subsistance au Magdalénien dans le Bassin de l'Aude. In: Bulletin de la Société préhistorique française, tome $96, \mathrm{n}^{\circ} 2,1999$. pp. 175-190;

doi : https://doi.org/10.3406/bspf.1999.10940

https://www.persee.fr/doc/bspf_0249-7638_1999_num_96_2_10940

Fichier pdf généré le 10/01/2019 


\begin{abstract}
Abstract While the Magdalenian population of the Aude basin is fairly well known nowadays, the subsistence strategies of the groups of humans had not yet been studied. Examination of the faunal remains from five sites (Lassac, Gazel, Canecaude and Belvis, excavated by D. Sacchi and La Grande Grotte at Bize, excavated by A. Tavoso) occupied between 17000 and 12000 BP has shown that the reindeer was the preferred game for 5000 years in the north of the Aude region (together with the varying hare at Gazel) - Fontána, 1998a. It has moreover revealed that all the northern sites were occupied (in the Badegoulian and Middle Magdalenian) during the same seasons (from late autumn to early spring) and that they were probably all living sites. Since only one period of the annual nomadic cycle was represented, I proposed that Ariège could correspond to the other part of the territory exploited by these groups. During the later Magdalenian, the sites (mainly in the south of the Pyrenean foothills) seem to testify to less extensive occupation and the Belvis cave was occupied in the summer months, probably intermittently, for hunting oriented towards ibexes, as part of a diversified spectrum. These data led me to envisage the hypothesis of greater mobility (during the summer months) in this region and for this period. Finally, research has shown that the possible seasonal movements of reindeer in no way resembled migrations and that the hypothesis of a temporary high altitude refuge after $12000 \mathrm{BP}$ (prior to the move towards the north) was not proved.
\end{abstract}

\title{
Résumé
}

Résumé Si le peuplement magdalénien du Bassin de l'Aude est aujourd'hui assez bien connu, les stratégies de subsistance des groupes humains n'avaient pas encore été étudiées. L'étude des restes fauniques de cinq sites (Lassac, Gazel, Cane- caude et Belvis, fouillés par D. Sacchi et La Grande Grotte de Bize, fouillée par A. Tavoso) occupés entre 17000 et 12000 B.P., a montré que le Renne avait été un gibier de prédilection durant 5000 ans dans le Nord de l'Aude (avec le Lièvre variable à Gazel) —Fontána, 1998a. De plus, elle a mis en évidence que tous les sites septentrionaux ont été occupés (au Badegoulien et au Magdalénien moyen) aux mêmes saisons (de la fin de l'automne au début du printemps) et qu'ils étaient probablement tous des sites résidentiels. Une seule partie du cycle annuel de nomadisme étant visible, j'ai proposé que l'Ariège pouvait constituer l'autre partie du territoire exploité. Au Magdalénien supérieur, les sites (majoritairement dans le sud des piémonts pyrénéens) semblent témoigner d'occupations de moindre étendue et la grotte de Belvis a été occupée à la belle saison probablement de façon discontinue avec une chasse orientée vers le Bouquetin, au sein d'un spectre diversifié. Ces données m'ont amenée à envisager l'hypothèse d'une plus grande mobilité (durant la belle saison) dans cette région et pour cette période. Enfin, l'étude a montré que les éventuels déplacements saisonniers du Renne ne ressemblaient en rien à des migrations et que I'hypothèse d'un refuge altitudinal temporaire après 12000 B.P. (avant le retrait vers le nord) n'était pas argumenté. 


\section{Mobilité et subsistance au Magdalénien dans le Bassin Laure FonTANa de l'Aude}

\begin{abstract}
Résumé
Si le peuplement magdalénien du Bassin de l'Aude est aujourd'hui assez bien connu, les stratégies de subsistance des groupes humains n'avaient pas encore été étudiées. L'étude des restes fauniques de cinq sites (Lassac, Gazel, Canecaude et Belvis, fouillés par D. Sacchi et La Grande Grotte de Bize, fouillée par A. Tavoso) occupés entre 17000 et 12000 B.P., a montré que le Renne avait été un gibier de prédilection durant 5000 ans dans le Nord de l'Aude (avec le Lièvre variable à Gazel) - Fontana, 1998a. De plus, elle a mis en évidence que tous les sites septentrionaux ont été occupés (au Badegoulien et au Magdalénien moyen) aux mêmes saisons (de la fin de l'automne au début du printemps) et qu'ils étaient probablement tous des sites résidentiels. Une seule partie du cycle annuel de nomadisme étant visible, j'ai proposé que l'A riège pouvait constituer l'autre partie du territoire exploité. Au Magdalénien supérieur, les sites (majoritairement dans le sud des piémonts pyrénéens) semblent témoigner d'occupations de moindre étendue et la grotte de Belvis a été occupée à la belle saison probablement de façon discontinue avec une chasse orientée vers le Bouquetin, au sein d'un spectre diversifié. Ces données m'ont amenée à envisager l'hypothèse d'une plus grande mobilité (durant la belle saison) dans cette région et pour cette période. Enfin, l'étude a montré que les éventuels déplacements saisonniers du Renne ne ressemblaient en rien à des migrations et que l'hypothèse d'un refuge altitudinal temporaire après 12000 B.P. (avant le retrait vers le nord) n'était pas argumenté.
\end{abstract}

\begin{abstract}
While the Magdalenian population of the Aude basin is fairly well known nowadays, the subsistence strategies of the groups of humans had not yet been studied. Examination of the faunal remains from five sites (Lassac, Gazel, Canecaude and Belvis, excavated by D. Sacchi and La Grande Grotte at Bize, excavated by A. Tavoso) occupied between 17000 and 12000 BP has shown that the reindeer was the preferred game for 5000 years in the north of the Aude region (together with the varying hare at Gazel) - Fontana, 1998a. It has moreover revealed that all the northern sites were occupied (in the Badegoulian and Middle Magdalenian) during the same seasons (from late autumn to early spring) and that they were probably all living sites. Since only one period of the annual nomadic cycle was represented, I proposed that Ariège could correspond to the other part of the territory exploited by these groups. During the later Magdalenian, the sites (mainly in the south of the Pyrenean foothills) seem to testify to less extensive occupation and the Belvis cave was occupied in the summer months, probably intermittently, for hunting oriented towards ibexes, as part of a diversified spectrum. These data led me to envisage the hypothesis of greater mobility (during the summer months) in this region and for this period. Finally, research has shown that the possible seasonal movements of reindeer in no way resembled migrations and that the hypothesis of a temporary high altitude refuge after 12000 BP (prior to the move towards the north) was not proved.
\end{abstract}




\section{PROBLÉMATIQUE ET MÉTHODE}

Dans quelle mesure l'étude de la grande faune mammalienne peut-elle contribuer à l'identification du système économique et du type de mobilité des Magdaléniens? Pour répondre à cette question, j'ai utilisé trois échelles d'analyse (fig. 1). À l'échelle des sites, il s'agissait d'identifier les comportements liés à l'alimentation carnée (choix des gibiers, stratégies d'acquisition, modalités de traitement et de consommation) et de déterminer les saisons de chasse. Les résultats obtenus contribuent à l'identification des modalités d'occupation des sites (fonction, saison et durée). $\grave{A}$ l'échelle microrégionale et régionale, il s'agissait de caractériser l'économie de chasse et d'identifier le cycle annuel de nomadisme régional, en comparant les données archéozoologiques de tous les sites. Les résultats contribuent à l'identification des modalités d'occupation du territoire exploité. À ce stade, la confrontation avec les autres données archéologiques est indispensable, notamment celles de l'origine des matières premières siliceuses et des coquillages.

$\grave{A}$ partir de cette conception du potentiel informatif des restes fauniques et de leur analyse intégrée, j'ai appréhendé l'organisation des groupes magdaléniens du Bassin de l'Aude en m'interrogeant sur leur comportement de subsistance, plus précisément:
- l'évolution des stratégies de subsistance et du peuplement, depuis le Badegoulien jusqu'au Magdalénien supérieur ;

- l'importance du Renne dans l'économie magdalénienne et le comportement de l'homme face à d'éventuelles migrations ;

- les modalités d'acquisition du gibier et d'autres denrées comme le silex et les coquillages: acquisitions intégrées ou spécialisées, acquisition directe ou par échange.

\section{LA RÉGION D'ÉTUDE, LES SITES ET LES FAUNES ÉTUDIÉES}

Quatre sites furent occupés au Badegoulien : la petite grotte de Bize, la grande grotte de Bize, Lassac et La Rivière (tabl. 1). Les cinq sites occupés au Magdalénien moyen sont les deux grottes de Bize, Canecaude, Gazel et La Crouzade ( $c f$. tabl. 1). Tous ces gisements se situent exclusivement dans le nord du Bassin de l'Aude, plus particulièrement sur les contreforts méridionaux de la Montagne Noire et près de la Méditerranée (fig. 2). Dix sites sont connus pour le Magdalénien supérieur; six se situent dans le sud : Fontlaurier, la Teulera, Les Conques, L'Eil, le Trou souffleur et Belvis et trois dans le nord: les grottes de Bize et la

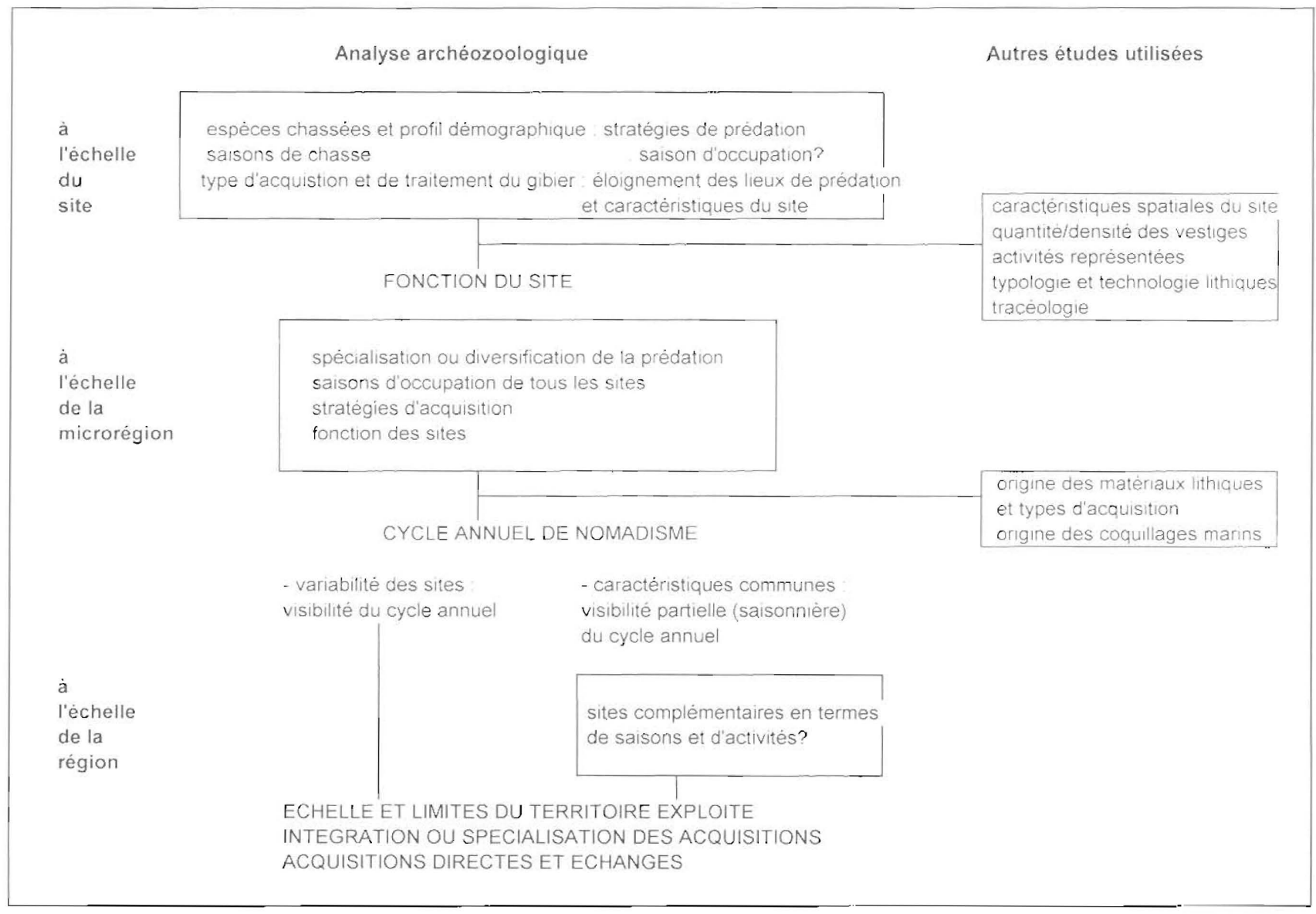

Fig. 1 - Problématique et méthode de l'étude. 


\begin{tabular}{|c|c|c|c|c|c|c|c|c|c|c|c|c|}
\hline Sites & Région & Altitude & Magdalénien & $\mathrm{C} 14$ & Stations & Travaux & Sup. max. & $\begin{array}{l}\text { Sup. } \\
\text { fouill. }\end{array}$ & $\begin{array}{l}\text { Ind. } \\
\text { lith NR }\end{array}$ & $\begin{array}{l}\text { Ind. } \\
\text { os. }\end{array}$ & Parure & $\begin{array}{l}\text { Faune } \\
\text { dét. }\end{array}$ \\
\hline Lassac & Montagne noire & $80 \mathrm{~m}$ & ancien & $x$ & Plein air & sondages & plus. hect & $6 \mathrm{~m}^{2}$ & 2000 & $x$ & & 250 \\
\hline Bize grande gr. & Montagne noire & $100 \mathrm{~m}$ & $\operatorname{arch} / \mathrm{myn} / \mathrm{sup}$. & $x$ & Grotte & fouilles & $1000 \mathrm{~m}^{2}$ & & ? & $x$ & $X$ & nombreux \\
\hline Bize petite gr. & Montagne noire & $100 \mathrm{~m}$ & anc/myn/sup. & & Grotte & fouilles & $250 \mathrm{~m}^{2}$ & & 940 & $X$ & $X$ & $?$ \\
\hline La Rivière & Montagne noire & $150 \mathrm{~m}$ & ancien & & Plein air & sondages & 10 ares & & 118 & & & rien \\
\hline Canecaude & Montagne noire & $250 \mathrm{~m}$ & moyen & $x$ & Grotte & fouilles & $100 \mathrm{~m}^{2}$ & $16 \mathrm{~m}^{2}$ & 5070 & $X$ & $x$ & 6769 \\
\hline Gazel & Montagne noire & $200 \mathrm{~m}$ & moyen & $X$ & Grotte & fouilles & $400 \mathrm{~m}^{2}$ & $20 \mathrm{~m}^{2}$ & $10000 ?$ & $x$ & $X$ & 16899 \\
\hline La Crouzade & Minervois & $50 \mathrm{~m}$ & moyen/sup. & & Grotte & fouilles & $400 \mathrm{~m}^{2}$ & & $?$ & $X$ & $X$ & ? \\
\hline Fontlaurier & Narbonnais & $?$ & Supérieur & & Plein air & fouilles & $?$ & & 10000 & & $X$ & $?$ \\
\hline Les Conques & Corbieres & $200 \mathrm{~m}$ & supérieur & $\bar{X}$ & Grotte & fouilles & $15 \mathrm{~m}^{2}$ & $9 \mathrm{~m}^{1}$ & 1000 & $\mathrm{X}$ & & 430 \\
\hline La Teulera & Corbières & $?$ & supérieur & & Plein air & sondages & $?$ & & 3500 & & & rien \\
\hline Le Harpon & Corbieres & $?$ & Supérieur & & Grotte & fouilles & $?$ & & $?$ & $?$ & & 3 \\
\hline L'CEil & Sud & $?$ & \begin{tabular}{|l|} 
supérieur \\
\end{tabular} & & Grolle & fouilles & $9 \mathrm{~m}^{2}$ & $9 \mathrm{~m}^{2}$ & 940 & $x$ & & très peu \\
\hline Le Trou souffleur & Conflent & $600 \mathrm{~m}$ & supérieur & & Grotte & sondages & $100 \mathrm{~m}^{2}$ & & 326 & $x$ & & très peu \\
\hline Belvis & Pays de Sault & $960 \mathrm{~m}$ & supérieur & $\mathrm{X}$ & Gronte & fouilles & $60 \mathrm{~m}^{2}$ & $30 \mathrm{~m}^{2}$ & $8000 ?$ & $x$ & $X$ & 2332 \\
\hline
\end{tabular}

Tabl. 1 - Caractéristiques des sites ct témoins archéologiques.

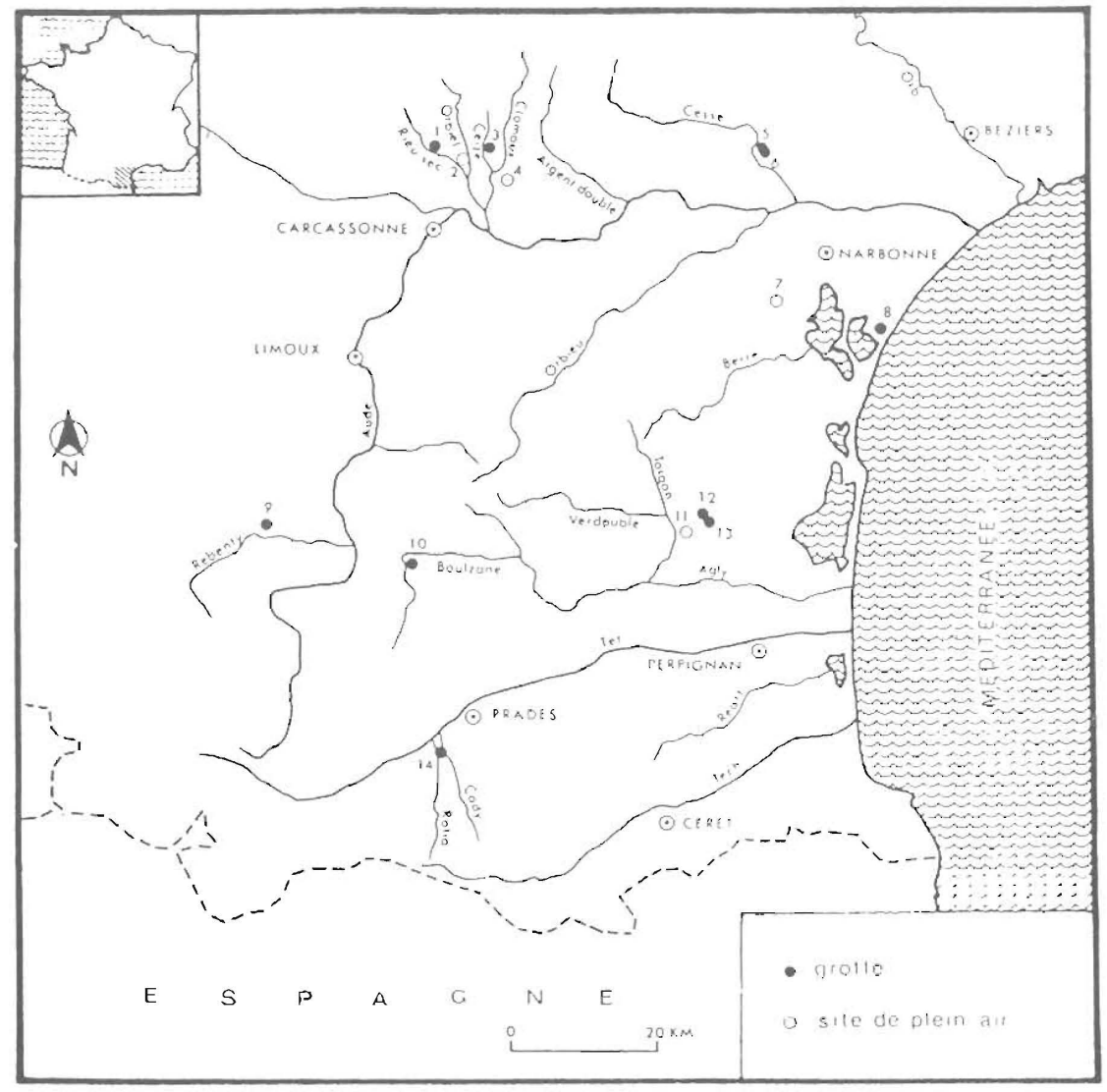

1: Canecaude (Aude);

2: Lassac;

3: Gazel ;

4: La Rivière ;

5: pelite grotte de Bize:

6: grande grotte de Bize;

7: Fontlaurier;

8: La Crouzade:

9: Belvis;

10: grotte de l'CEil:

11 : La Teulera (Pyrénées orientales);

12: Les Conques;

13: Le Harpon:

14: Le Trou souffleur.

Fig. 2 - Carte des sites magdaléniens du Bassin de l'Aude.

Crouzade ( $c f$. tabl. 1 et fig. 2). J'ai étudié quatre collections issues de sites récemment fouillés par D. Sacchi : Lassac pour le Badegoulien, Canecaude et Gazel pour le Magdalénien moyen et Belvis pour le Magdalénien supérieur. J'ai également étudié les restes dentaires de Renne du niveau badegoulien de la grande grotte de Bize (issus des fouilles Tavoso) et j'ai utilisé les données préliminaires (Tavoso, 1987) de ce site pour le Badegoulien et le Magdalénien supérieur (étudié actuellement par M. Patou-Mathis) et celles des Conques pour le Magdalénien supérieur (Moigne et al., 1998).
Ces collections fauniques sont numériquement différentes (cf. tabl. 1): plus de 200000 restes à Gazel, environ 45000 à Canecaude, 32000 à Belvis et 355 à Lassac.

\section{Les espèces représentées}

Les taxons représentés dans les spectres fauniques de Lassac, de Canecaude, de Gazel et de Belvis sont identiques à ceux d'autres sites magdaléniens des Pyrénées ou du sud-ouest (Clottes, 1989 ; Delpech, 1983) : grands bovinés, équidés, Bouquetin, Chamois, Renne, Cerf, 


\begin{tabular}{|l|l|c|c|c|c|}
\cline { 3 - 6 } \multicolumn{1}{l|}{} & Gazel & $\begin{array}{l}\text { Cane- } \\
\text { caude }\end{array}$ & Belvis & Lassac \\
\hline Loutre & Lutra lutra & $\mathrm{X}$ & & & \\
\hline Lynx & Lynx lynx & $\mathrm{X}$ & & & \\
\hline $\begin{array}{l}\text { Hyène des } \\
\text { cavernes }\end{array}$ & Crocuta crocura & $\mathrm{X}$ & $\mathrm{X}$ & & \\
\hline Loup & Canis lupus & $\mathrm{X}$ & $\mathrm{X}$ & & \\
\hline Ours & Ursus sp. & $\mathrm{X}$ & $\mathrm{X}$ & $\mathrm{X}$ & \\
\hline Renard & $\begin{array}{l}\text { Vulpes vulpes/ } \\
\text { Alopex lagopus }\end{array}$ & $\mathrm{X}$ & $\mathrm{X}$ & $\mathrm{X}$ & \\
\hline Sanglier & Sus scrofa & $\mathrm{X}$ & $\mathrm{X}$ & $\mathrm{X}$ & \\
\hline Lapin & $\begin{array}{l}\text { Oryctolagus } \\
\text { cuniculus }\end{array}$ & $\mathrm{X}$ & $\mathrm{X}$ & $\mathrm{X}$ & \\
\hline Lièvre variable & Lepus timidus & $\mathrm{X}$ & $\mathrm{X}$ & $\mathrm{X}$ & \\
\hline Cheval & Equus gallicus & $\mathrm{X}$ & $\mathrm{X}$ & $\mathrm{X}$ & \\
\hline Isard & $\begin{array}{l}\text { Rupicapra } \\
\text { pyrenaica }\end{array}$ & $\mathrm{X}$ & $\mathrm{X}$ & $\mathrm{X}$ & \\
\hline Cerf & Cervus elaphus & $\mathrm{X}$ & $\mathrm{X}$ & $\mathrm{X}$ & \\
\hline Bison/Aurochs & Bison/Bos & $\mathrm{X}$ & $\mathrm{X}$ & $\mathrm{X}$ & $\mathrm{X}$ \\
\hline Bouquetin & Capra pyrenaica & $\mathrm{X}$ & $\mathrm{X}$ & $\mathrm{X}$ & $\mathrm{X}$ \\
\hline Renne & $\begin{array}{l}\text { Rangifer } \\
\text { tarandus }\end{array}$ & $\mathrm{X}$ & $\mathrm{X}$ & $\mathrm{X}$ & $\mathrm{X}$ \\
\hline & NR déterminés & $\mathrm{16} 899$ & 6769 & 2332 & 252 \\
\hline & Nombre de taxons & $\mathrm{15}$ & 13 & $\mathrm{11}$ & 3 \\
\hline
\end{tabular}

Tabl. 2 - Espèces représentées dans les sites étudiés.

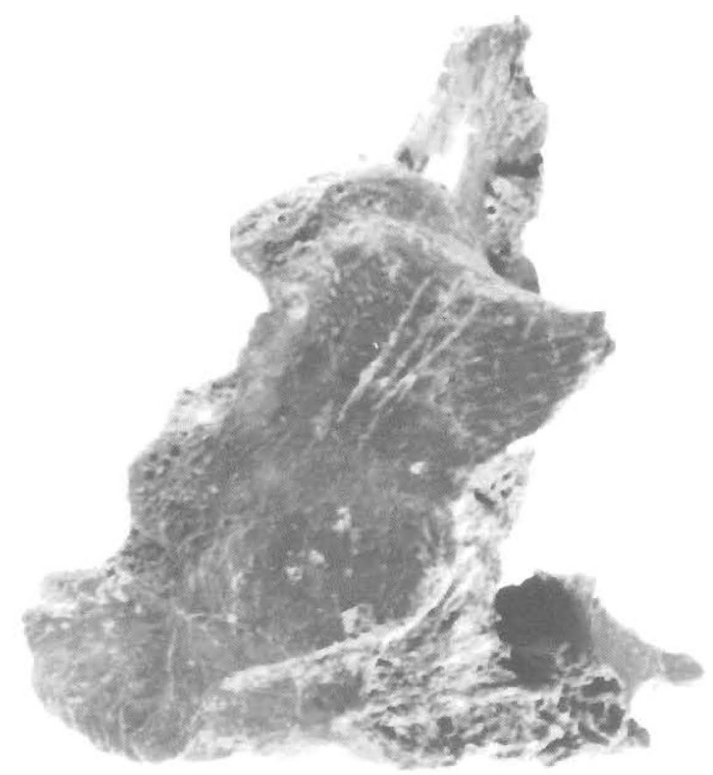

Fig. 3 - Traces d'enlèvement de la peau sur un fragment de maxillaire de Lynx (Gazel, C7).

Sanglier, carnivores et lagomorphes. Seuls le Chevreuil et l'Antilope saïga sont absents (tabl. 2). Oiseaux et Poissons ont été identifiés et déterminés à Gazel, à Canecaude et à Belvis (Desse-Berset, inédit; Le Gall, dans Sacchi, 1994 ; Le Gall, 1999 ; Vilette, 1983 et dans Sacchi, 1994). Le nombre de taxons est étroitement corrélé au nombre de restes déterminés, et les données de Lassac ont été considérées comme peu représentatives (site de plein air estimé à plusieurs hectares et dont les fouilles n'ont concerné qu'une dizaine de mètres carrés). Dans les autres sites audois, les mêmes espèces sont identifiées.

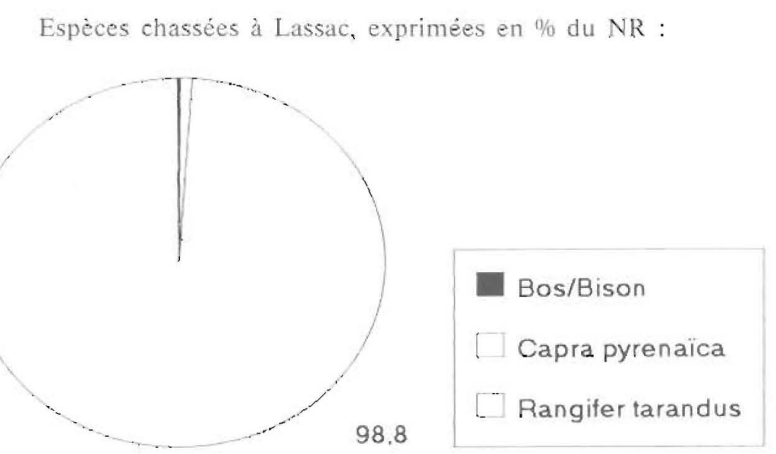

Représentation des espèces chassées à Canecaude. exprimée en \% du NR:

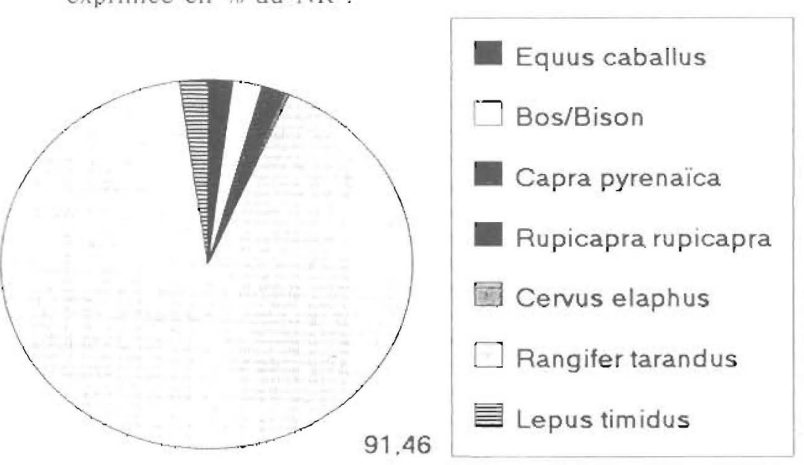

Données fauniques préliminaires de la grande grotte de Bize (fouilles Tasovo, étude en cours M. Patou-Mathis):

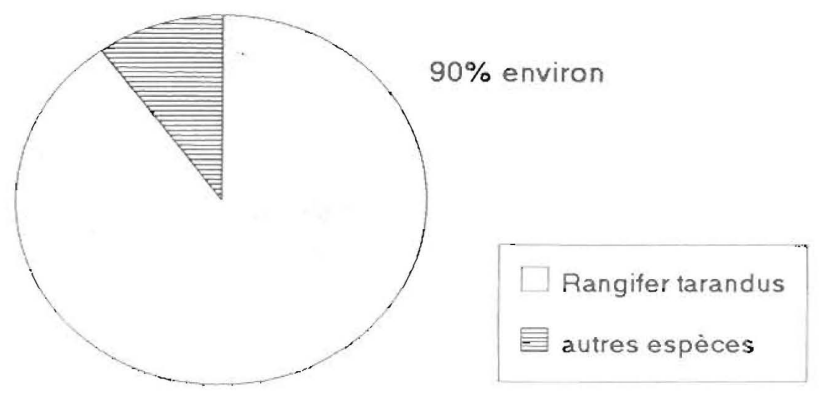

Fig. 4-Représentation quantitative des espèces chassées à Bize, à Lassac et à Canecaude.

J'ai tenté de distinguer les espèces acquises par l'Homme de celles dont l'origine est naturelle. Si j'ai conclu au statut de gibier pour les lièvres variables des trois sites et le Lynx de Gazel (tabl. 3) en raison de la présence de traces d'outils lithiques (fig. 3), celui des autres espèces reste plus sujet à caution et je ne les ai pas considérées comme des espèces chassées en l'absence de preuves (autres carnivores, Lapin, Sanglier, certains oiseaux). Les espèces acquises par l'Homme sont donc au nombre minimum de sept à Canecaude et à Belvis, de huit à Gazel avec le Lynx, et seulement de trois à Lassac. Si on excepte ce cas, la richesse est peu différente d'un site à l'autre.

Au-delà de cette ressemblance en termes qualitatifs, la représentation quantitative de ces gibiers diffère selon les sites. Les deux spectres badegouliens (de Lassac et de Bize) et celui du Magdalénien moyen de Canecaude montrent l'existence d'une chasse spécialisée au Renne (fig. 4), alors qu'à Gazel le Lièvre variable fut tout 
autant recherché (fig. 5). Le choix préférentiel de ce lagomorphe (86 individus) n'a pas été mis en évidence sur d'autres sites magdaléniens d'Europe occidentale. À la période suivante, le Renne reste le gibier de prédilection dans cette région (à Bize) : la place de ce gibier est donc prédominante dans l'alimentation des Magdaléniens du nord de l'Aude, quelle que soit la période considérée et le spectre du niveau Magdalénien supérieur de la grande grotte de Bize ne semble pas montrer une éventuelle diversification de la chasse. À Belvis, si une seule espèce, le Bouquetin, domine le spectre faunique, sa représentation ne dépasse pas $67 \%$ du NR et $35 \%$ du NMIc (fig. 6). Les autres gibiers sont représentés de façon beaucoup plus équilibrée que dans les autres sites: entre 6 et 7 individus pour le Chamois, le Cerf, le Lièvre variable et le Cheval. La diversité est ici la plus forte de notre corpus et parler de chasse spécialisée au Bouquetin nous semblerait abusif (16 individus sur 46). Néanmoins ce seul spectre ne peut argumenter la diversification de la chasse vers 12000 B.P. d'autant que les autres ressources (poissons et oiseaux) sont très marginales et que l'altitude de Belvis $(1000 \mathrm{~m})$ en fait un site particulier. Dans cette région du sud, le Renne est encore chassé avec le Bouquetin à proximité du site des Conques (Moigne et al., 1998) alors qu'il est rarissime à Belvis (deux restes).

\section{Modalités de traitement du gibier}

Les gibiers ont-ils été apportés entiers ou en partie sur les sites? Leur destination finale a-t-elle toujours été la à Gazel, exprimée en $\%$ du N.R. :
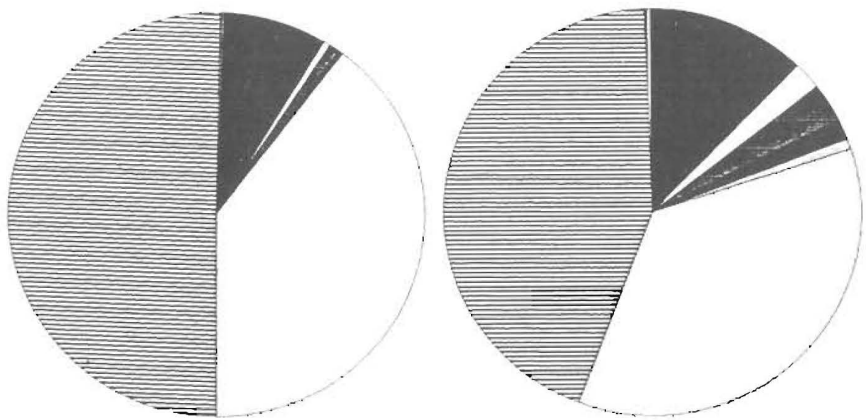

Fig. 5 - Représentation quantitative des espèces chassées à Gazel.

Représentation des espèces chassées à Belvis, exprimée en $\%$ du N.R.

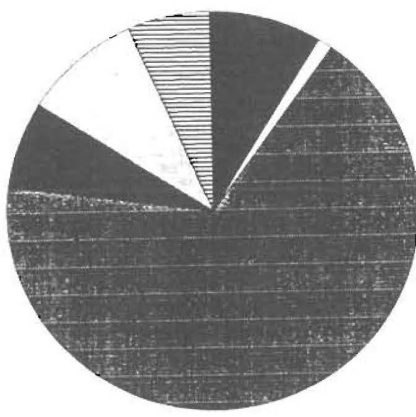

Représentation des espèces chassėes à Belvis, exprimée en $\%$ du N.M.I.f.

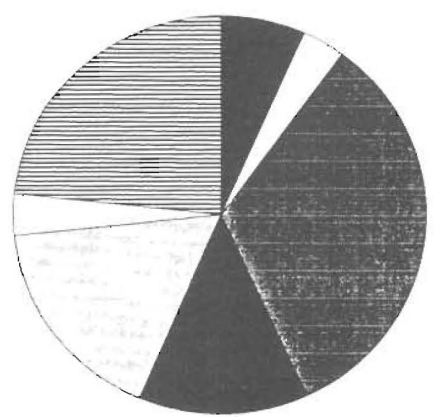

consommation sur le site ou certaines parties ont-elles ensuite été emportées ailleurs (et sous quelle forme?). Cet aspect est essentiel dans cette étude puisqu'il contribue à la détermination de la fonction des sites. Cette analyse est fondée sur l'interprétation de la représentation des parties du squelette et j'ai tenté de distinguer ce qui, dans ces représentations, relève de la conservation différentielle, de la fragmentation (détermination) différentielle et de l'action anthropique, pour les principaux gibiers.

\section{Les rennes de Gazel et de Canecaude}

Toutes les parties du squelette sont représentées (fig. 7), ce qui montre que certains animaux sinon tous ont été apportés entiers sur le site: ceci suggère la proximité du lieu de chasse. Néanmoins, le degré de corrélation entre le pourcentage des parties observées et le pourcentage de survie attendu (d'après les modèles de Binford, 1978) diffère selon les os (fig. 8). J'ai attribué la sous représentation des vertèbres, des côtes et du coxal à la fragmentation différentielle (200000 esquilles indéterminées à Gazel) tandis que celle de la phalange 3 peut s'expliquer si on imagine qu'elle est restée attachée à la peau lors de l'enlèvement de cette dernière. Seules les sous représentations de la scapula et de l'humérus ne nous semblent pas pouvoir s'expliquer par le facteur de fragmentation, d'autant que le radius est bien représenté. Les seuls os sur-représentés sont la mandibule ainsi que les métapodes à Canecaude et dans une moindre mesure à Gazel. Les traces attestant l'enlèvement de la peau, la

Représentation des espèces chassées à Gazel, exprimée en $\%$ du N.M.I.c.

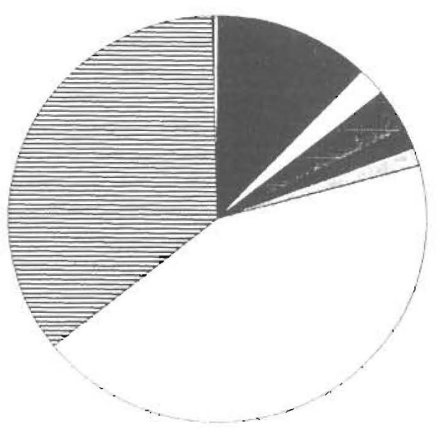

Equus caballus Bos/Bison

Capra pyrenaïca

Rupicapra rupicapra $\square$ Cervus elaphus [ Rangifer tarandus 砉 Lepus timidus [IIIII) Lynx lynx
Représentation des espèces chassées à Belvis, exprimée en \% du N.M.I.c. :

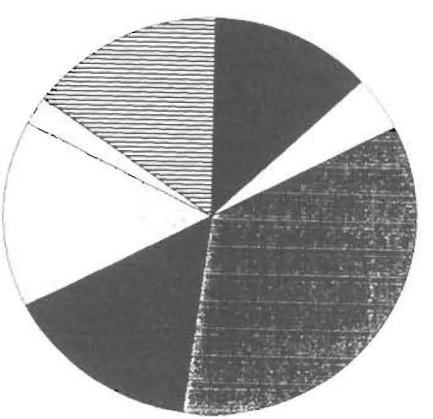

Equus caballus Bos/Bison

Capra pyrenaíca

Rupicapra rupicapra

$\square$ Cervus elaphus

Rangifer tarandus

Lepus timidus

Fig. 6 - Représentation quantitative des espèces chassées à Belvis 


\begin{tabular}{|l|r|r|r|r|r|r|r|}
\cline { 2 - 8 } \multicolumn{1}{c|}{} & \multicolumn{1}{c|}{ NR } & $\%$ NR & NMIf & $\%$ NMIf & NMIc & $\%$ NMIC \\
\hline \multicolumn{7}{|c|}{ CANECAUDE } \\
\hline Equus caballus & 131 & 2 & 7 & 7,4 & 10 & 6,1 \\
\hline Bos/Bison & 148 & 2,3 & 4 & 4,2 & 7 & 4,2 \\
\hline Capra pyrenaïca & 57 & 0,9 & 4 & 4,2 & 7 & 4,2 \\
\hline Rupicapra pyrenaïca & 72 & 1,1 & 6 & 6,3 & 12 & 7,3 \\
\hline Cervus elaphus & 13 & 0,2 & 1 & 1,1 & 1 & 0,6 \\
\hline Rangifer tarandus & 5878 & 91,5 & 69 & 72,6 & 124 & 75,2 \\
\hline Lepus timidus & 128 & 2 & 4 & 4,2 & 4 & 2,4 \\
\hline Total déterminés & 6427 & & 95 & & 165 & \\
\hline
\end{tabular}

\begin{tabular}{|l|r|r|r|r|r|r|r|}
\cline { 2 - 7 } \multicolumn{1}{c|}{} & NR & $\%$ NR & NMIf & $\%$ NMIi & NMIc & $\%$ NMIc \\
\hline \multicolumn{7}{|c|}{ GAZEL } \\
\hline Equus caballus & 1366 & 8,2 & 24 & 12,5 & 29 & 12,2 \\
\hline Bos/Bison & 94 & 0,6 & 4 & 2,1 & 5 & 2,1 \\
\hline Capra pyrenaïca & 157 & 0,9 & 6 & 3,1 & 8 & 3,3 \\
\hline Rupicapra pyrenaïca & 17 & 0,1 & 3 & 1,6 & 4 & 1,7 \\
\hline Cervus elaphus & 20 & 0,1 & 2 & 1 & 3 & 1,3 \\
\hline Rangifer tarandus & 6647 & 39,7 & 69 & 35,7 & 104 & 43,7 \\
\hline Lepus timidus & 8460 & 50,5 & 84 & 43,5 & 84 & 35,3 \\
\hline Lynx lynx & 1 & 0,01 & 1 & 0,5 & 1 & 0,4 \\
\hline Total déterminés & 16762 & & 193 & & 238 & \\
\hline
\end{tabular}

\begin{tabular}{|l|r|r|r|r|r|r|r|}
\cline { 2 - 8 } \multicolumn{1}{c|}{} & NR & NR & NMIf & M N NIf & NMIc & NMIC \\
\hline Equus caballus & 205 & 9,1 & 2 & 6,67 & 6 & 13 \\
\hline Bos/Bison & 23 & 1,02 & 1 & 3,33 & 2 & 4,4 \\
\hline Capra pyrenaica & 1512 & 67,11 & 10 & 33,33 & 16 & 34,8 \\
\hline Rupicapra rupicapra & 158 & 7,01 & 4 & 13,33 & 7 & 15,2 \\
\hline Cervus elaphus & 213 & 9,46 & 5 & 16,68 & 7 & 15,2 \\
\hline Rangifer tarandus & 2 & 0,09 & 1 & 3,33 & 1 & 2,2 \\
\hline Lepus timidus & 140 & 6,21 & 7 & 23,33 & 7 & 15,2 \\
\hline Total déterminés & 2253 & & 30 & & $\mathbf{4 6}$ & \\
\hline
\end{tabular}

\begin{tabular}{|l|r|r|}
\cline { 2 - 3 } \multicolumn{1}{c|}{} & \multicolumn{1}{c|}{ NR } & $\%$ NR \\
\hline \multicolumn{2}{|c|}{ LASSAC } \\
\hline Bos/Bison & 1 & 0,4 \\
\hline Capra pyrenaïca & 2 & 0,8 \\
\hline Rangifer tarandus & 248 & 98,8 \\
\hline Total déterminés & 251 & \\
\hline
\end{tabular}

Tabl. 3 - Especes chassées dans les sites magdaléniens audois.

désarticulation et le décharnement sont également semblables sur les deux sites et démontrent la dissociation complète des os des membres tout en séparant le membre antérieur en deux tronçons : scapula-humérus d'une part et radius d'autre part qui confirme les taux de représentation différentiels (fig. 9). Le mode de découpe du rachis n'a pu être mis en évidence et notamment la séparation éventuelle des côtes des vertèbres. L'idée d'un stockage de viande sous cette forme n'apparaît donc pas comme évidente d'autant que la sous représentation des vertèbres semble d'abord liée à la fragmentation. Il semble que la récupération de la moelle et celle des tendons sur les métapodes ait été systématique (type de fragmentation et traces caractéristiques).

Les rennes ont donc été apportés entiers sur les deux sites et consommés au moins en partie sur place. Si certains ont peut-être été éviscérés sur le lieu de chasse cela ne fut pas la règle puisque certains os de fœtus ont été identifiés. La distorsion tête/squelette post-crânien ne nous semble pas être uniquement redevable au facteur de détermination différentielle mais peut être également dû à un comportement humain : rejet hors site de certains os, ou transport de certaines parties charnues avec les os, voire stockage sous cette forme?

\section{Les lièvres variables de Gazel}

Les lièvres variables de Gazel ont également été apportés entiers et rejetés sur le site, les seules sous représentations concernant les os les plus petits du squelette et les os sujets à l'écrasement comme le crâne, les côtes, le sternum et certaines vertèbres (fig. 10). Toutes les autres parties sont représentées de façon homogène, par tronçons laissant deviner le mode de désarticulation. Cette cohérence montre également que la conservation des parties squelettiques de cet animal n'est pas différentielle comme dans le cas des grands herbivores.

La répartition spatiale des restes, leur taux de représentation et les quelques traces d'outils lithiques attestent de certaines désarticulations (fig. 11): extrémités des membres (parfois en connexion), rachis détaché avec les côtes, membres probablement désolidarisés.

Les bouquetins de Belvis (fig. 12)

Si les déficits et les absences (sternum, sacrum, atlas et axis) sont beaucoup plus marqués, j'ai beaucoup insisté sur le fort taux de fragmentation à Belvis, qui a rendu la détermination très délicate et produit un nombre d'indéterminés important, certainement parmi les os plats et les vertèbres. Les autres os sont bien représentés et on constate par ailleurs une bonne concordance fémur-tibia, phalanges 1-2, humérus-radius, os hyoïde-mandibule, ce qui atteste de l'apport et du rejet sur le site de ces os ensemble. Cette fois, la représentation de la mandibule n'est donc pas la seule à être forte. Par conséquent, si des modalités de rejet différentiel peuvent expliquer les différences de pourcentages entre les os longs, il semble néanmoins que les bouquetins ont été apportés entiers et consommés sur le site (en grande majorité). Le mode de découpe n'a pas été mis en évidence dans le détail en raison du mauvais état de surface des os et de la faible quantité d'os anatomiquement déterminés portant des traces. La récupération de la moelle et des tendons semble avoir été systématique.

\section{Stratégies de chasse et saisonnalité}

La sélection des espèces s'est-elle accompagnée de la sélection de certains individus en terme d'âge et de sexe traduisant ainsi une stratégie de chasse précise ? Et durant quelles saisons les gibiers ont-ils été chassés ? À partir de la détermination de la composition démographique des groupes d'herbivores les mieux représentés, j'ai construit des profils de mortalité. À partir du classement des dents de Renne en cinq classes d'âge (0-1/1-3/ 3-6/6-10/plus de 10), j'ai comptabilisé les individus. Deux pourcentages ont été calculés, à partir du nombre de dents et du NMI de combinaison. L'amplitude des 


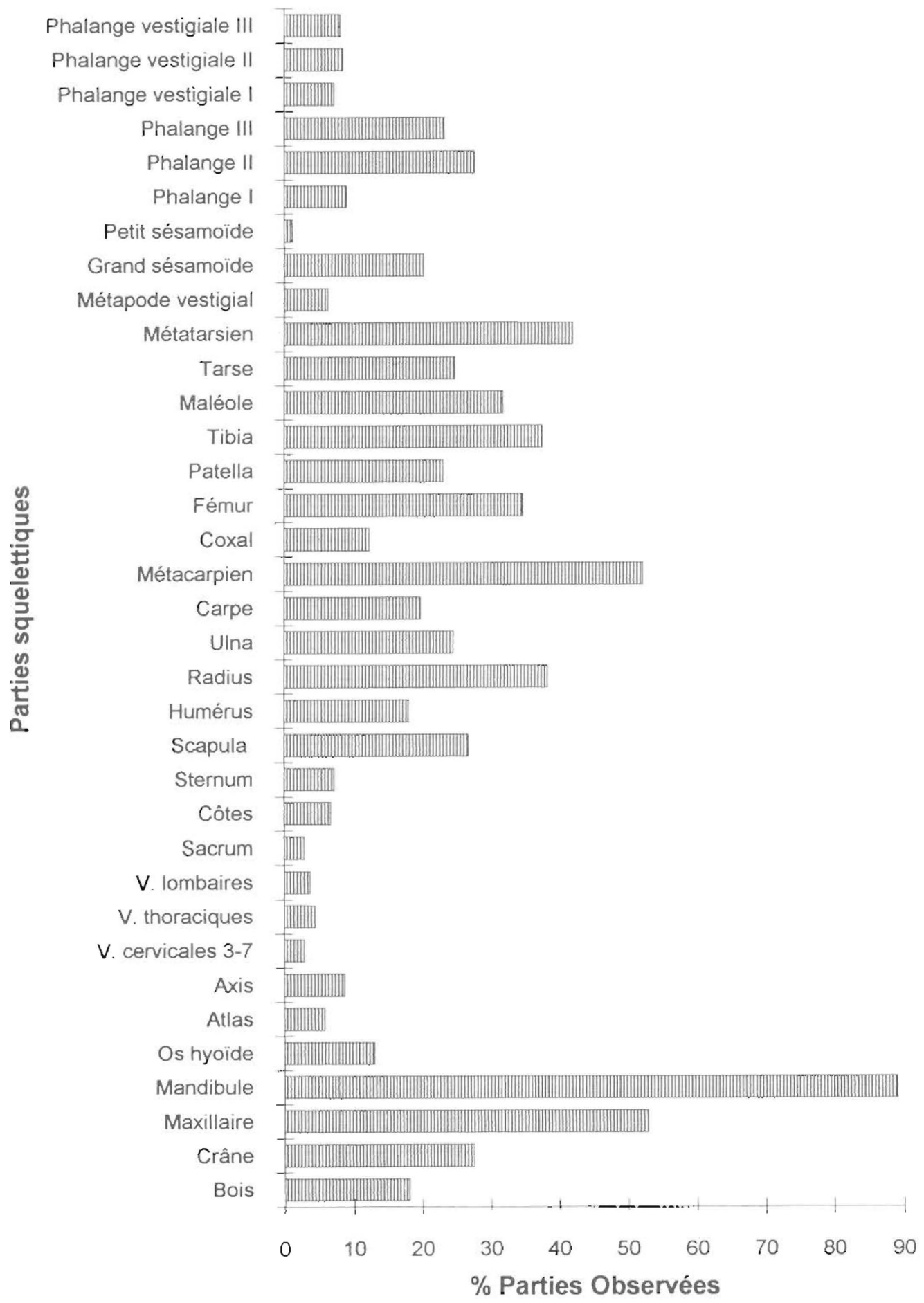

Fig. 7 - Représentation des parties squelettiques des rennes de Gazel.

classes n'étant pas la même (un an pour la $1^{\text {re }}$, deux ans pour la $2^{\mathrm{i}}$, trois ans pour la $3^{\mathrm{c}}$, quatre ans pour la $4^{\mathrm{e}}$ et huit ans pour la $5^{\mathrm{e}}$ ), j'ai pondéré les effectifs à l'intérieur de chaque classe, afin d'obtenir des pourcentages à signification égale. Pour interpréter les profils obtenus, j'ai utilisé les données d'une population de référence, les caribous Kaminuriak du Canada, étudiés par F. Miller (1974) et G. Parker (1972). Il s'agit d'un corpus photographique de mandibules d'individus de tous âges et des deux sexes, associé à une description de l'évolution dentaire. J'ai également utilisé les données de l'éthologie fournies par ces deux études pour interpréter mes résultats. Si j’ai déterminé les saisons en fonction des dates d'éruption et du degré d'usure pour les rennes des trois premières années, je n'ai pu évaluer la saison de mort des adultes par cette méthode. C'est 


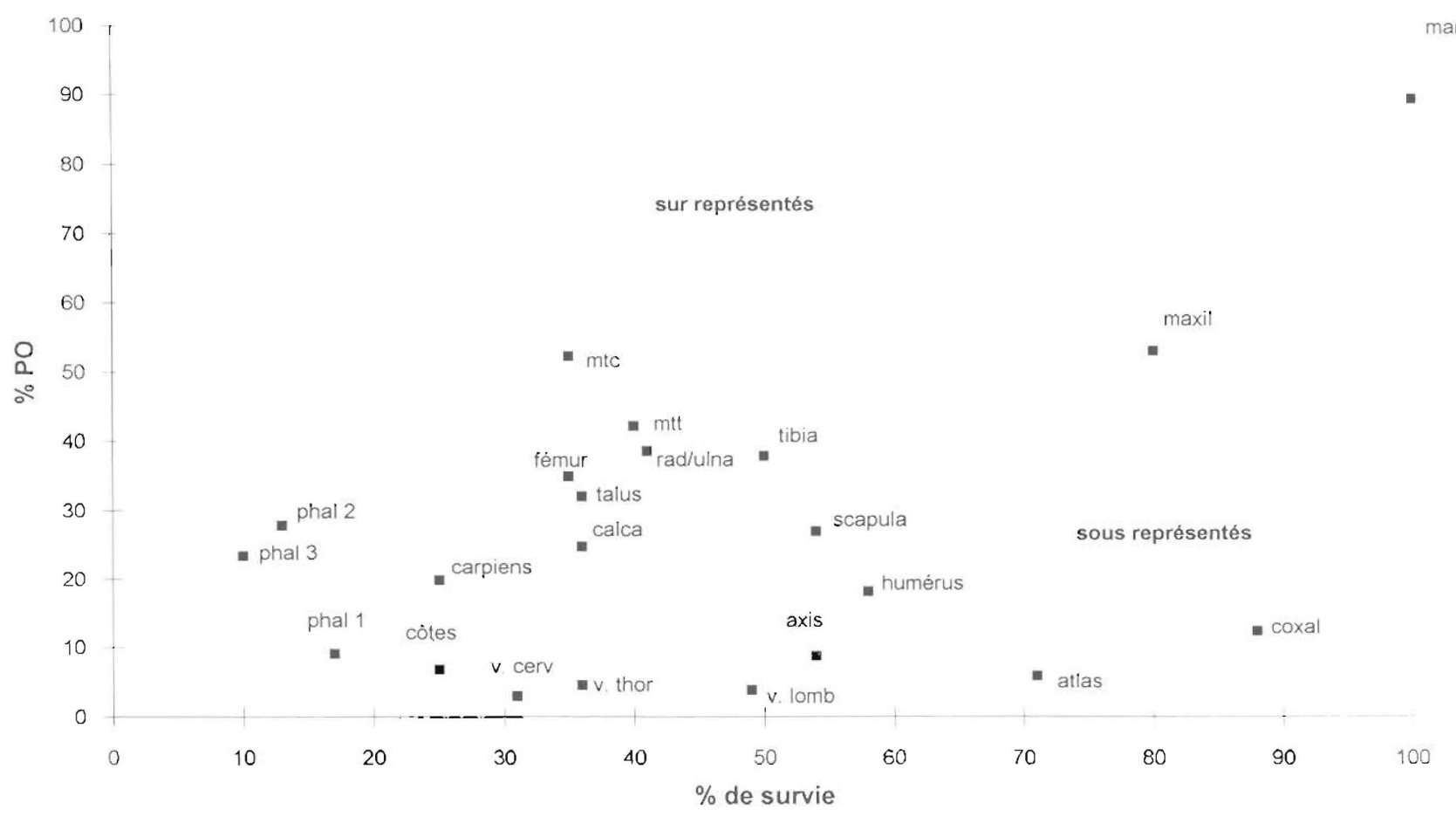

Fig. 8 - Relation entre le pourcentage des parties observées et le pourcentage de survie pour les rennes de Gazel.

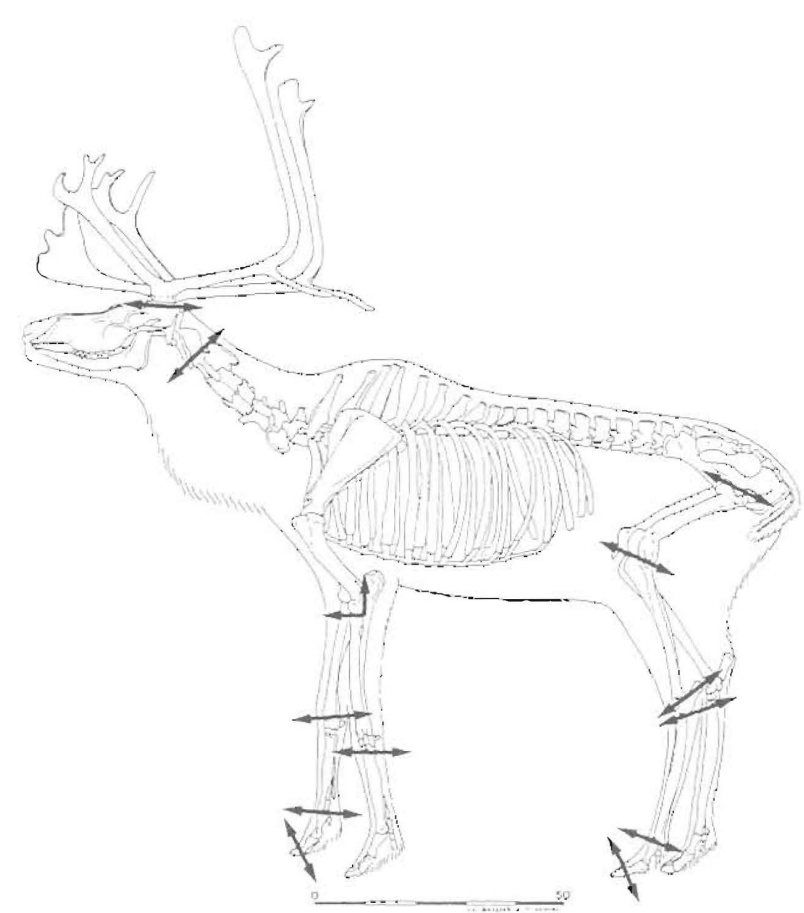

Fig. 9 - Localisation des principales traces de découpe des rennes de Canecaude et de Gazel.

par déduction et en confrontant les données des appendices frontaux et de l'éthologie que j'ai pu déterminer les saisons de chasse au Renne adulte. Si cette étude est fondée principalement sur l'analyse des restes dentaires, elle s'appuie en effet sur l'étude des bois de Renne à Gazel, à Canecaude et à la grande grotte de Bize. Pour les jeunes rennes de la première année, la taille de la dague indique très bien l'âge, permettant d'en déduire la saison et jusqu'à trois ans, son diamètre permet une détermination fiable si elle est entière (Bouchud, 1966). Si les bois de cervidés permettent donc une première évaluation de la saison, les pièces caractéristiques et mesurables sont le plus souvent rares dans les sites : ce matériau ayant avant tout servi de matière première à une industrie développée, il est en grande majorité fragmenté (seuls les déchets nous parviennent) ou totalement transformé (les produits finis).

L'étude des restes dentaires de Renne et dans une moindre mesure celle des bois, a mis en évidence la récurrence d'une même stratégie dans les quatre sites de la Montagne Noire: les deux sites du Badegoulien (Lassac et Bize) et les deux du Magdalénien moyen (Canecaude et Gazel, fig. 13). En effet, les profils obtenus correspondent à celui d'une population vivante de rennes de toundra (Miller, 1974). Ces profils de type catastrophiste représentent donc une population de rennes que les chasseurs ont pris pour cible sans distinction d'âge. De plus, l'étude des rennes de la première année a montré, sur les quatre sites, qu'aucun individu de moins de 6-8 mois n'a été tué, absence qui n'est pas imputable au facteur de conservation différentielle (présence de nombreuses dents lactéales isolées, cartilages intercostaux conservés, par exemple). S'il apparaît que les rennes de la première année ont été chassés entre les mois de novembre et d'avril (mise bas mai-juin), l'examen des dents des jeunes de la seconde et de la troisième année a montré cette même absence d'animaux abattus entre le printemps et le début de l'automne. Ce résultat a été corroboré par l'étude des bois de Renne de Canecaude et de Gazel : les fragments conservés sont des bois de massacre de femelles 


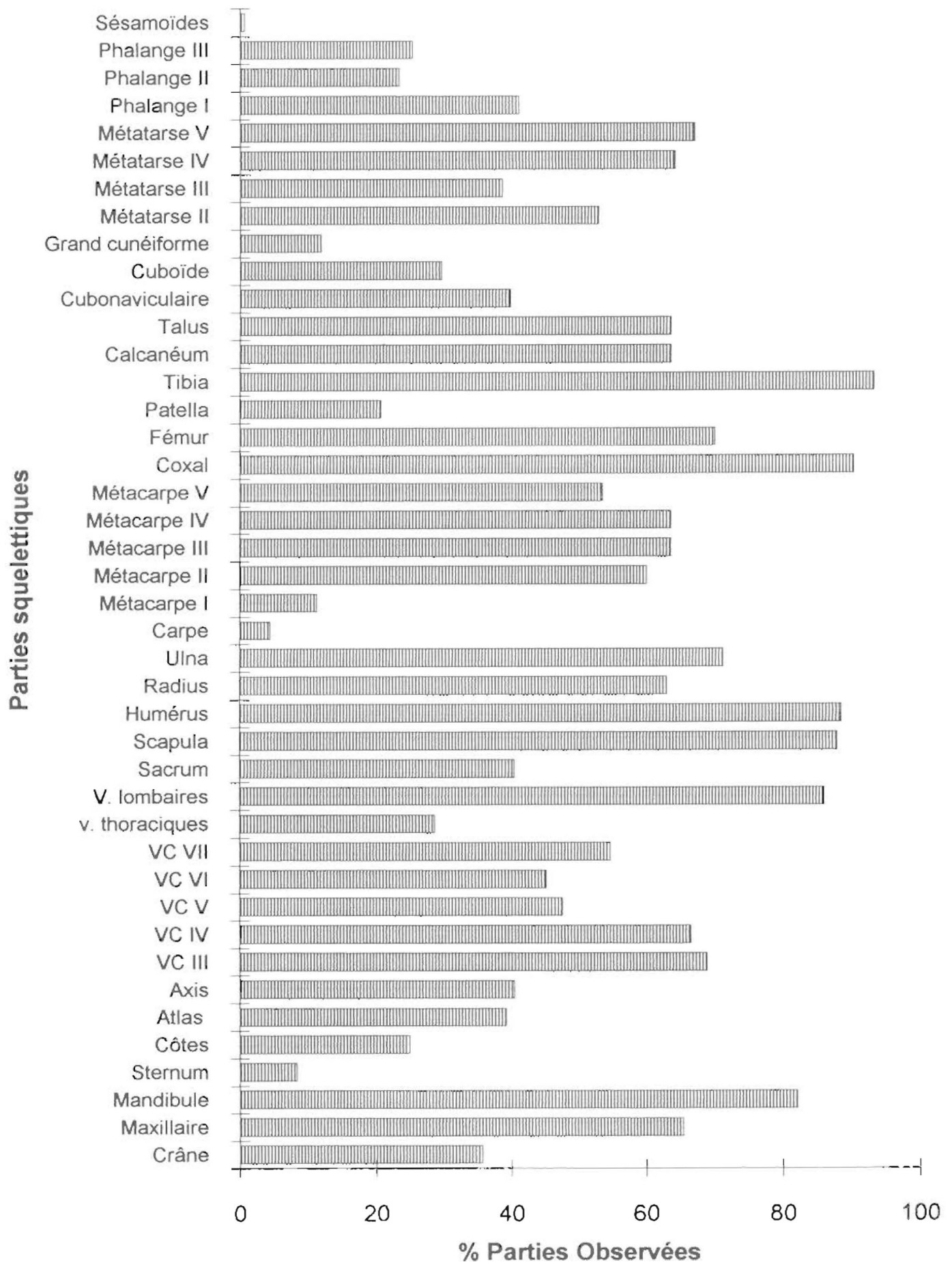

Fig. 10 - Représentation des parties squelettiques des lièvres variables de Gazel.

adultes (dont la ligne de mue qui apparaît à la fin de l'hiver (Bouchud, 1966) est bien visible sur certains bois), jeunes et sub-adultes ( 25 fragments déterminés à Canecaude et 13 à Gazel). Seul un bois de mâle adulte a été identifié à Canecaude. Cette composition démographique (femelles adultes et jeunes) correspond au noyau hivernal d'un grand troupeau de rennes composé de femelles adultes et de jeunes, les mâles adultes restant isolés et parfois éloignés de $100 \mathrm{~km}$ (Parker, 1972). Les chasseurs magdaléniens ont donc pris pour cible ces rennes rassemblés sans distinction d'âge ni de sexe, durant six mois environ, de la fin de l'automne au début du printemps.

Les résultat obtenus sur la saisonnalité de la chasse à certains autres herbivores et sur la pêche (Le Gall, 1999; Desse-Berset, inédit) ont confirmé les 


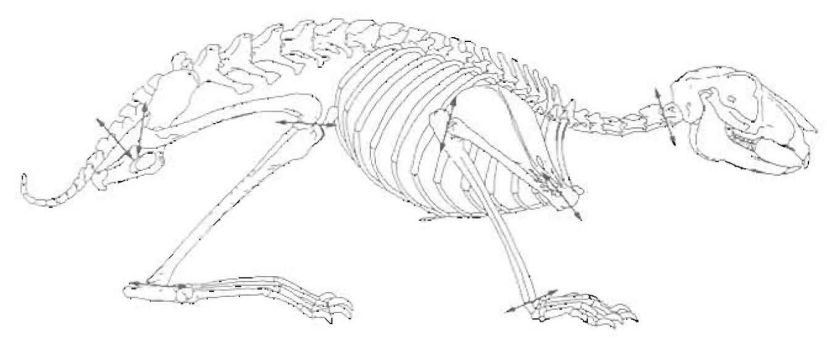

Fig. 11 - Localisation des principales traces de découpe et de désarticulation des lièvres variables de Gazel.

résultats obtenus pour le Renne. Tout d'abord, les profils de survie pour les autres taxons (grands bovinés, Chamois, Bouquetin et Cerf) sont sensiblement identiques à ceux du Renne, à l'exception de celui du Cheval.
J'ai construit les histogrammes de chasse en NMIc après les déterminations des âges à partir de divers référentiels (tabl. 4). Seules les dents ont permis ces déterminations, les appendices frontaux étant rares et fragmentés. Les données numériques sont très inférieures à celles du Renne, le nombre des individus représentés dans les histogrammes n'excédant dix que dans un seul cas. La détermination de la saison a été effectuée sur très peu de restes (et d'individus) à chaque fois, et on ne peut exclure que d'autres saisons soient représentées. L'analyse des profils est basée, pour le Cheval, sur les travaux de M.-A. Levine 1983, et sur les travaux plus généraux pour les autres espèces (Stiner, 1990 ; Caughley, 1966; Lyman, 1987).

À Canecaude, des histogrammes de chasse ont été construits pour le Bouquetin, les bovinés, les équidés et l'Isard, seuls les deux derniers groupes ayant produit

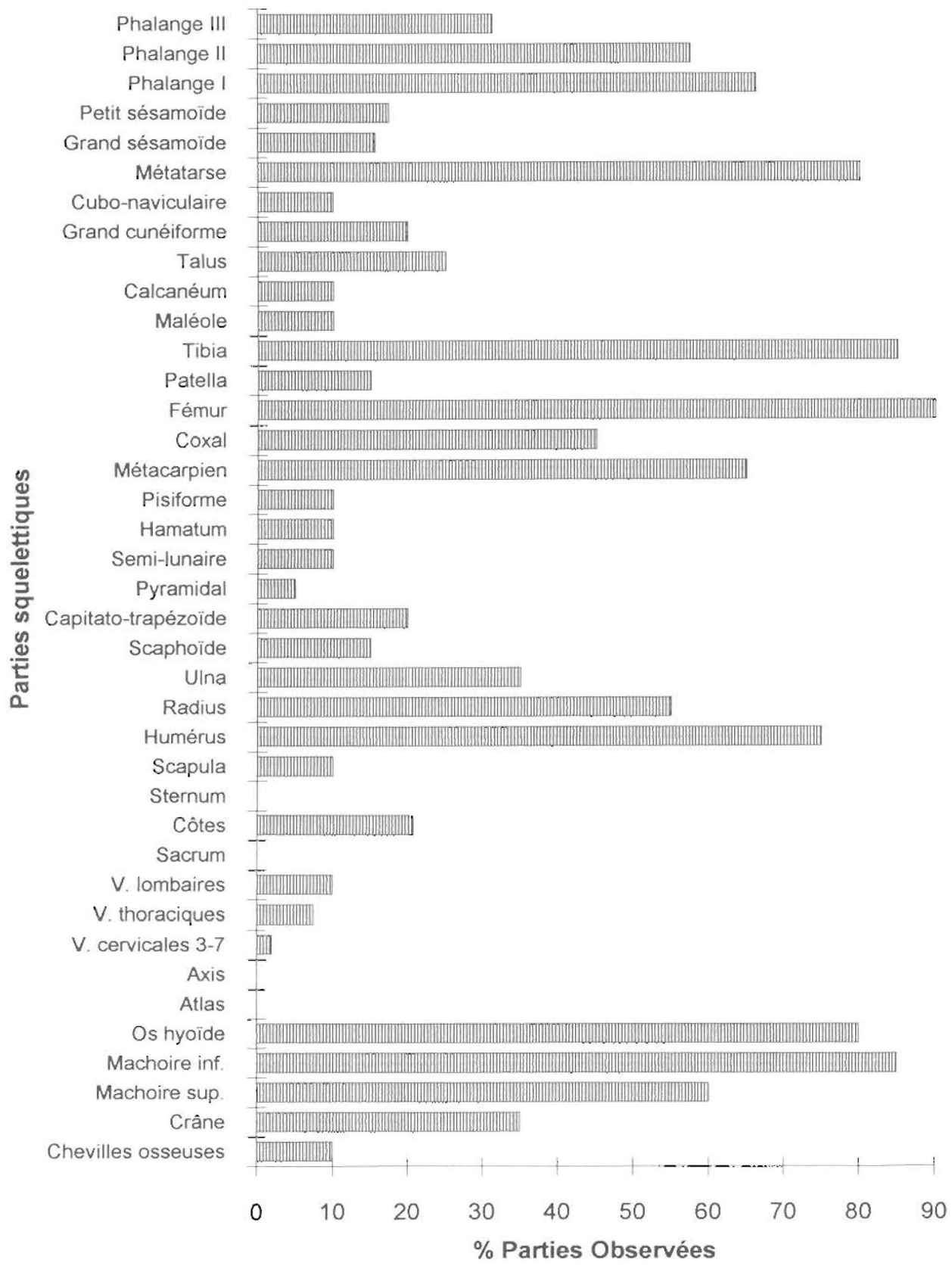

Fig. 12 - Représentation des parties squeleltiques des bouquetins de Belvis. 


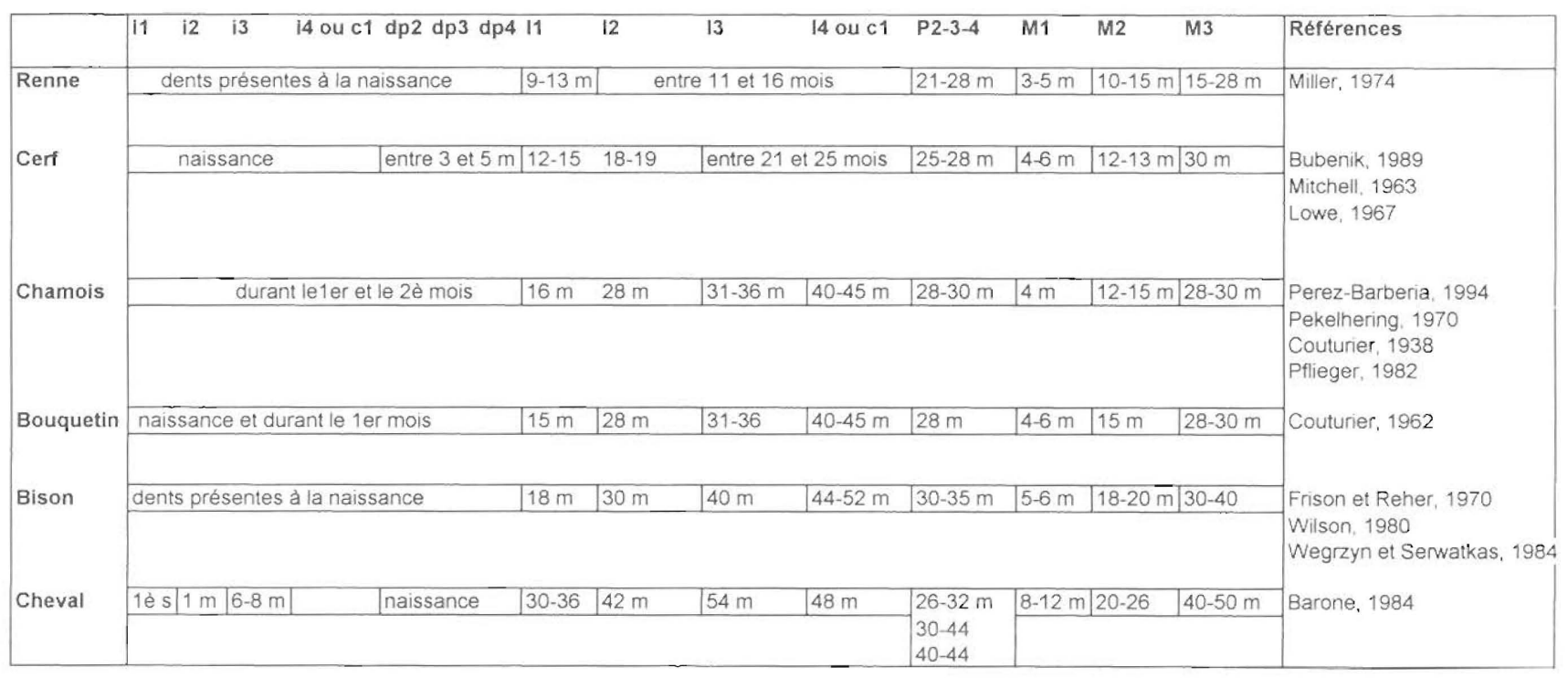

Tabl. 4 - Datcs d'éruption de dents jugales de certains herbivores.

des résultats de saisonnalité. La période de chasse des isards âgés de moins de trois ans (7) a été déterminée grâce à l'usure des $4^{\text {es }}$ prémolaires déciduales dont trois appartiennent à des animaux tués au printemps et quatre (dont le plus jeune) entre le mois d'octobre et la fin de l'hiver. Pour les équidés (10 individus), deux individus de la première année sont représentés par trois incisives lactéales dont l'usure permet d'attribuer (de façon approximative) à ces deux animaux un âge situé entre 6 mois et un an, donc une chasse d'automne ou d'hiver. Les données de saisonnalité concernant la pêche (Le Gall, 1992 et 1999) mentionnent une capture des truites en début de belle saison et des saumons en fin de bonne saison, pour les quelques individus comptabilisés. Ces données semblent donc confirmer une occupation de Canecaude entre les mois d'octobre et de mars environ et il semble que les isards et les équidés (comme le Renne) aient été chassés tout au long de cette période, à la différence des poissons.
On ne peut évidemment assurer que bouquetins et bovinés n'ont pas été tués hors de ces périodes.

À Gazel, seules trois espèces ont fait l'objet de déterminations d'âge mais les indications de saisonnalité sont plus nombreuses et quelques données concernant le sex-ratio ont pu être obtenues. Toutes les classes d'âge sont représentées chez les équidés ( 29 individus) et les données de saisonnalité, obtenues à partir de onze prémolaires déciduales (4 individus) montrent deux épisodes de chasse, un en automne, et un au printemps. Parmi les 9 bouquetins, j'ai déterminé les saisons de chasse de trois individus de la première année, à partir de quatre prémolaires déciduales: le printemps pour deux (un mois et un an), et l'automne pour un (six-huit mois). Deux épisodes de chasse sont donc représentés. Le seul boviné dont on puisse déterminer la saison de mort est un jeune de la première année, tué en automne ou au début de l'hiver. Les trois cerfs chassés sont des individus âgés de 1 à 4 ans et le plus jeune a pu

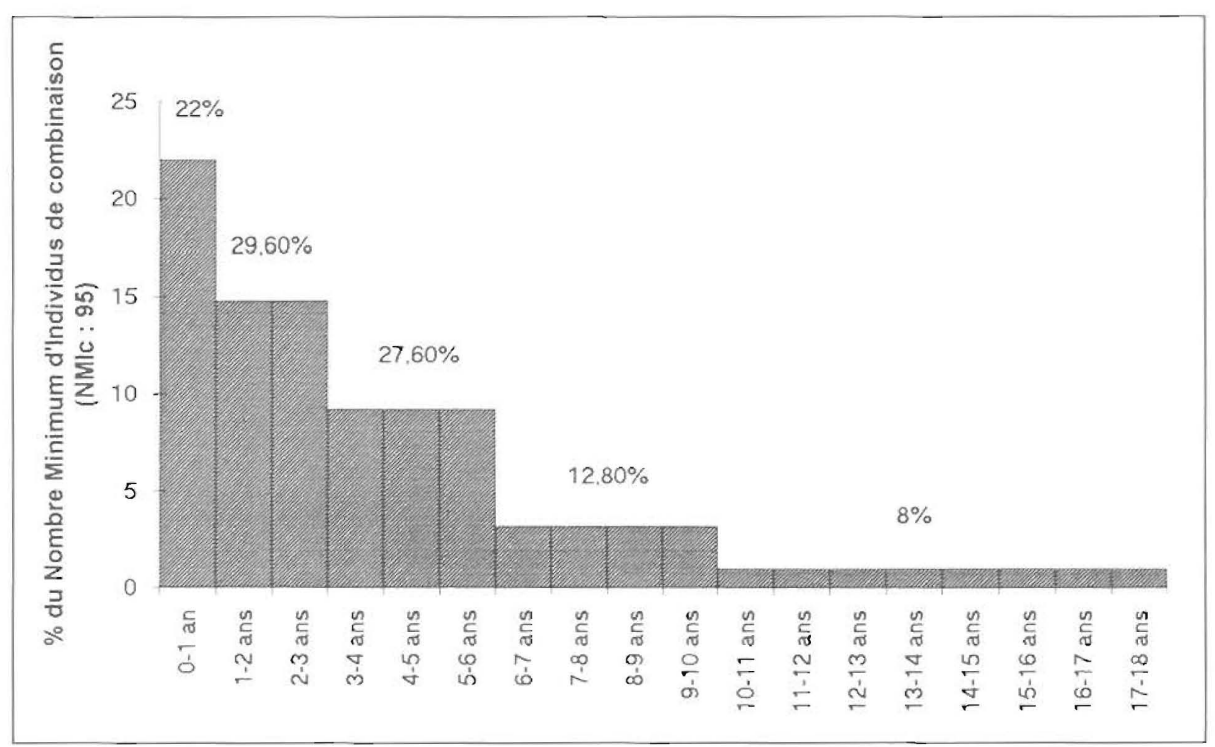

Fig. 13 - Âge d'abattage des rennes de Gazel. 
ÂGE DES BOUQUETINS

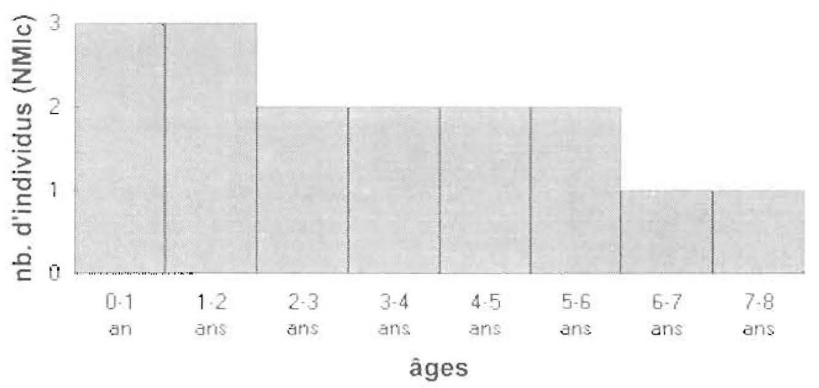

ÂGE DES ISARDS

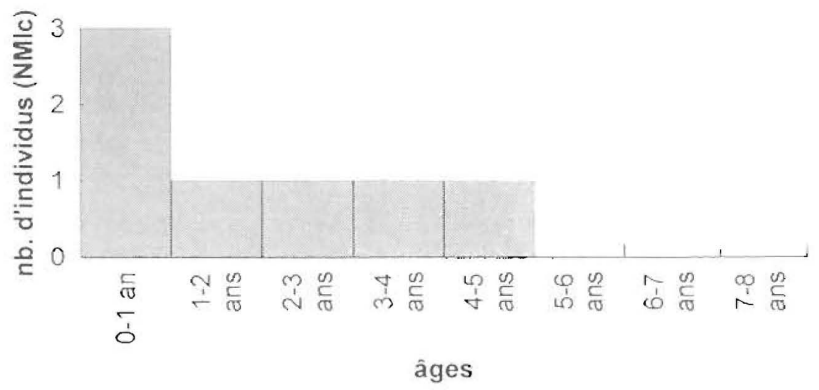

Fig. 14 - Âge d'abattage des bouquetins et des isards de Belvis.

être abattu au printemps (bourgeons de seconde incisive définitive). Seul le Renne a été chassé de façon continue de l'automne au printemps. Les Poissons (truites, saumons et cyprinidés) ont probablement été pêchés à la fin de l'hiver ou au début du printemps (Desse-Berset, inédit).

À Belvis, j'ai déterminé l'âge des quatre espèces les mieux représentées (Bouquetin, Cerf, Cheval, Isard), seuls les équidés ne fournissant pas d'indications de saison. Les trois bouquetins âgés de moins d'un an ont probablement été tués chacun à une saison différente, seule la saison d'hiver n'étant pas représentée. Pour les isards la saison de chasse est identique à celle du
ÂGE DES CERFS


âges

Fig. 15 - Āge d'abattage des cerfs et des équidés de Belvis.

Bouquetin, les trois individus de moins d'un an ayant été tués, chacun à une saison différente hors de l'hiver. Les deux cerfs de la première année ont été tués, l'un en été, l'autre au printemps. Les poissons ont été pêchés, selon O. Le Gall, durant "toute la belle saison" (dans Sacchi, 1994). Certains bouquetins et isards ont donc été chassés du printemps au début de l'automne, certains cerfs au printemps et en été alors que la période de pêche pourrait s'étendre du mois de mars au mois d'octobre. Il est délicat de conclure à la sélection d'individus à partir d'échantillons si faibles, mais les profils des cerfs et des isards ressemblent plutôt à des profils de survie, alors qu'il est plus difficile de trancher pour les autres (fig. 14 et fig. 15).

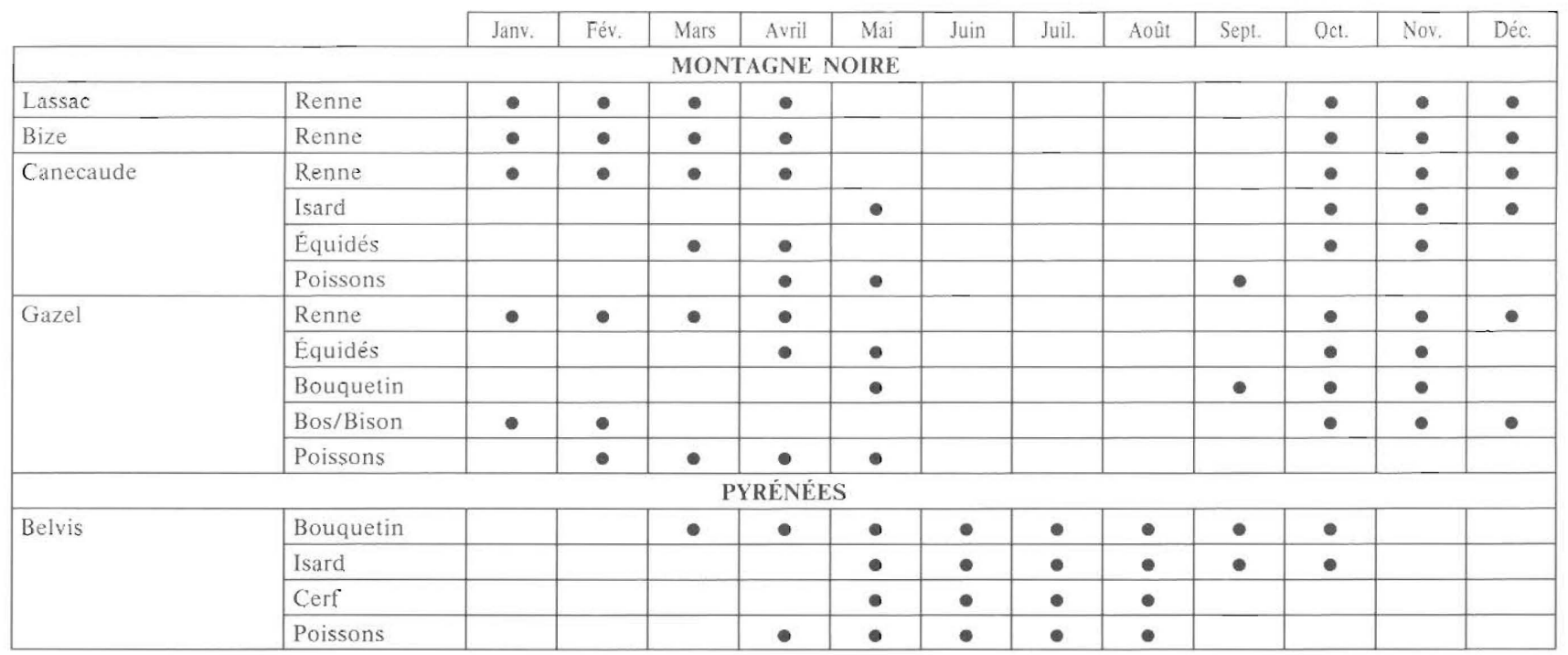

Tabl. 5 - Saisons de chasse aux grands herbivores et de pêche sur les sites magdaléniens audois. 
Ces trois sites présentent trois cas de figure différents du point de vue des épisodes de chasse et de leur continuité (tabl. 5). À Gazel, les hommes ont chassé le Renne de façon continue durant six mois entre octobre et mai. Ils ont chassé en outre surtout les équidés, au printemps et en automne seulement, et le Bouquetin, l'Isard, et le Cerf. Il est très regrettable qu'on ne puisse déterminer la saison de chasse du Lièvre variable: on ne peut que supposer qu'étant donné l'importance de lièvres tués, leur capture a pu avoir lieu en hiver, saison où sa fourrure est la plus fournie. À Canecaude, le Renne fut également chassé de la fin de lautomne au début du printemps, continuellement comme les autres gibiers, à la différence de Gazel. La chasse a donc été plus diversifiée sur toute la période, à Canecaude, d'une façon toute relative étant donné la proportion de Renne. Cette continuité apparente peut recouvrir trois réalités différentes: une seule occupation continue de six mois, plusieurs occupations successives (de quelques semaines) et discontinues au cours des six mois, ou plusieurs occupations d'un mois par exemple, chaque année. Il semble que les données stratigraphiques ne puissent pas distinguer une fréquentation unique d'occupations multiples. La récurrence de cette période de six mois sur quatre sites nous incite à penser qu'il s'agit d'occupations continues. De plus, un argument lié à la comparaison ethnographique nous porte à le croire. Même si nous avons dit plus haut qu'une période d'occupation continue de cinq mois n'était pas documentée chez les populations actuelles du Nord, il reste vrai que l'hiver est la période de rassemblement des groupes Eskimos sur des sites importants, pour trois mois d'hiver. De plus, nous savons que les rennes de la Montagne Noire n'ont pas été chassés en masse lors d'une migration, mais tout au long de l'hiver, en raison de leur présence prolongée. Cette permanence des rennes expliquerait donc ainsi la possibilité d'un séjour de six mois des hommes, par rapport aux populations actuelles. À Belvis, si le Bouquetin et l'Isard ont été chassés à trois saisons différentes, hors hiver, la continuité de ces chasses n'est pas prouvée. Ce site aurait donc été fréquenté à plusieurs saisons à l'échelle d'une année (les hommes seraient revenus périodiquement) ou bien à une saison (ou moins) par année.

Cette idée de discontinuité de l'occupation nous semble confirmée par l'étude aviaire de $\mathrm{Ph}$. Vilette (1983). En effet, après avoir envisagé l'exploitation de certains oiseaux pour les plumes, il pense que certains rapaces ont également niché dans la grotte, et il conclut: "Dans ce cas et si des oiseaux de proies ont niché au niveau de la grotte, on peut supposer que c'était en l'absence de l'homme qui serait donc arrivé après la nidification, c'est-à-dire à partir de ce qui correspond, de nos jours, à la fin du mois de Juillet. D'autres données fauniques indiquent pourtant une occupation du site pendant tout l'été." Nos données n'indiquent pas une chasse durant les trois mois d'été, et confirment ainsi l'hypothèse de $\mathrm{Ph}$. Vilette : une chasse aux oiseaux à la fin de l'été. L'identification de l'oiseau gravé sur un fragment d'os comme un oiseau migrateur avait poussé M. Crémades (1994) à identifier une saison qui ne peut être cependant que celle à laquelle l'oiseau est vu (et non celle de l'occupation du site).

\section{STRATÉGIES D'ACQUISITION, HABITAT ET MOBILITÉ DES GROUPES MAGDALÉNIENS DE L'AUDE}

\section{Au Badegoulien et au Magdalénien moyen}

J'ai tenté de déterminer la fonction des sites en mettant en relation les activités attestées, les stratégies de chasse, la durée et la saison d'occupation, afin de mettre en évidence la totalité du cycle annuel de nomadisme. J'ai également mis ces données en perspective avec celles relatives à l'approvisionnement en matière première siliceuses et en coquillages (lieux d'origine, mode d'acquisition) afin de proposer un type d'organisation des acquisitions et de la mobilité.

Il semble que quatre des six gisements de la Montagne Noire (Lassac, grande grotte de Bize, Gazel et Canecaude) représentent des sites résidentiels d'hiver, occupés environ six mois. Il n'est pas possible de conclure pour les autres occupations des grottes de Bize, de La Crouzade et de la Rivière, les deux derniers sites étant vraisemblablement des établissements tout aussi importants. Aucun d'entre eux ne semble avoir été occupé à la belle saison et aucun site d'acquisition spécialisée de courte durée (silex ou gibier) n'est identifié: il semble donc qu'une seule partie du cycle annuel de nomadisme soit représentée dans l'Aude. Avant de rechercher l'éventuelle trace de ces groupes durant la belle saison, j'ai pris connaissance de l'origine des matériaux allochtones présents dans ces sites et j'ai envisagé plusieurs hypothèses pour expliquer cette absence apparente d'occupation de l'Aude durant la moitié de l'année dont une qui m'a paru plus convaincante : un déplacement de tous les groupes à la belle saison en direction des proches Pyrénées. La présence de sites ariégeois au Magdalénien moyen complémentaires en terme de saison (Enlène - Lalande, 1986 -, Le Mas d'Azil en partie - Patou, 1984 et 1985 -) montre que les Magdaléniens qui passaient l'hiver en Montagne Noire auraient pu passer la belle saison en Ariège. L'acquisition du silex aurait-elle été spécialisée, effectuée par de petits groupes durant l'hiver? Les Magdaléniens audois auraient-ils pu échanger en Ariège des coquillages méditerranéens voire des fourrures de lièvres contre des coquillages atlantiques et peut-être d'autres produits comme de la parure ou des objets d'art mobilier? La chasse au Renne pouvait toujours être pratiquée comme l'attestent les spectres fauniques des sites ariégeois. Dans cette hypothèse, la particularité de ces groupes audois tiendrait pour une part à l'exploitation du Renne, peut-être tout au long de l'année, et d'autre part à un cycle annuel fondé sur l'échange de produits de la chasse (peaux, fourrures), coquillages atlantiques et méditerranéens. Enfin, si l'occupation de Canecaude (CII : (Gif 2708) $14230 \pm$ 160 B.P.) - Sacchi, 1986 - a pu être partiellement contemporaine de celle d'Enlène - Bégouen et Clottes, $1981-(C 3:$ [Gif 4124] $13940 \pm 250$ B.P.), il n'en est pas 
de même pour celle de Gazel dont l'occupation - C7 : ([Gif 2655] $15070 \pm 270$ B.P.) - (Sacchi, 1986) est la plus ancienne des sites pyrénéens du Magdalénien moyen et elle n'a donc pas actuellement d'équivalent en Ariège.

Enfin, la question de la présence supposée du Renne durant six mois dans le Nord de l'Aude m'a engagée à étudier celle des migrations de Renne à la fin du paléolithique supérieur. Cette persistance des rennes femelles avec les jeunes dans une même microrégion confirment les données de certains sites du Sud-Ouest et des Pyrénées où les rennes ont été chassés la moitié de l'année voire plus. Si les rennes migraient au Würm IV dans cette région, je me suis demandé quelle avait été l'étendue de leurs déplacements et surtout leur direction.

Mon analyse métrique des populations chassées de Canecaude et de Gazel a montré que ces rennes étaient plus petits que ceux des deux sites landais Dufaure et Duruthy (les différences significatives concernent les métapodes). Mais l'analyse de la comparaison de la taille des rennes de l'Aude et des Landes est restée délicate puisqu'elle ne s'est fondée que sur quatre sites couvrant une séquence chronologique large (de 15000 à 11000 B.P. environ) et qu'elle a croisé trois paramètres (le sex ratio, l'appartenance à une population géographique, l'évolution éventuelle de la taille pendant 4000 ans dans chacune de ces deux régions). Néanmoins, si les rennes de l'Aude semblent plus petits que ceux des Landes, l'hypothèse de l'appartenance à deux populations différentes reste envisageable, en raison de deux arguments, si fragiles soientils : une éventuelle stabilité de la taille des rennes aux abords du Massif pyrénéen et des périodes de chasses identiques dans les sites considérés (donc de sex ratio assez proches). Si tel était le cas, l'hypothèse de migrations le long de la chaîne pyrénéenne ne pourrait être retenue, mais plutôt celle de migrations très restreintes, peut-être altitudinale en direction des Pyrénées ou des contreforts méridionaux du Massif Central, restreintes car un mois avant la mise bas et à peine un mois après le rut, les femelles et les jeunes sont déjà ou encore en Montagne Noire (Fontana, 1995). Néanmoins, la possibilité d'une sédentarité des rennes dans l'Aude ne peut être écartée. Enfin, si les corpus du Magdalénien moyen ne sont pas assez conséquents dans les Pyrénées, celui du Magdalénien supérieur de Bize (étude M. Patou-Mathis) contribuera à cette problématique.

\section{Au Magdalénien supérieur}

L'économie de chasse des Magdaléniens audois de cette période reste plutôt mal connue, comme l'acquisition des autres produits de subsistance et la saisonnalité des occupations, aussi bien au nord qu'au sud. Il est donc encore impossible d'entrevoir le cycle annuel de nomadisme et de discerner le type de mobilité des Magdaléniens. Dans le nord, seules les données de la grande grotte de Bize montrent la persistance de la chasse spécialisée au Renne. Dans le sud, je n'ai pu déterminer si Belvis était partie intégrante d'un territoire pyrénéen qui s'étendait des Pyrénées Orientales jusqu'en Ariège. La préférence pour le Bouquetin, dans les sites de Belvis et des Conques est en effet observable dans les Pyrénées ariégeoises sur les sites de La Vache (Pailhaugue, 1996), des Églises (Delpech et Le Gall, 1983; Delpech et Villa, 1993), de Rhodes II (Delpech, 1983) et de Bédeilhac (Bahn, 1983). Tous ces gisements sont situés à l'Est du Bassin de Tarascon, dans un rayon de $50 \mathrm{~km}$, à des altitudes comprises entre 520 et $960 \mathrm{~m}$ (à l'exception des Conques) et j'ai montré que la complémentarité saisonnière et fonctionnelle de ces sites était envisageable (Fontana, 1998c).

Enfin, l'hypothèse d'un refuge altitudinal temporaire du Renne dans les Pyrénées avant son retrait vers le Nord de l'Europe, semble difficile à soutenir au regard des données des sites pyrénéens sensu lato. La présence du Renne dans l'Aude et dans les Pyrénées-Orientales en altitude au Magdalénien supérieur est limitée à un seul site, Belvis (deux restes) vers 12270 B.P. alors qu'il est abondant à des altitudes moins élevées, aux Conques. Le seul site d'altitude $(1000 \mathrm{~m})$ plus récent est la Balma Margineda (sur la frontière espagnole, à 60 $\mathrm{km}$ à vol d'oiseau de Belvis) dont le niveau azilien (10 $640 \pm 260$ B.P.) ne contient aucun reste de Renne (Geddes et al., 1985 et 1986). De même, de l'autre côté de la chaîne pyrénéenne, dans la couche azilienne (II) du site de Zatoya (Altuna et Mariezkurrena, 1996) qui se situe à une altitude proche de $1000 \mathrm{~m}$, aucun reste de Renne n'a été déterminé (comme dans tous les autres sites cantabriques à cette période) alors qu'il était présent au Magdalénien supérieur/final. Les seuls sites de l'extrême fin du Magdalénien où le Renne abonde sont des sites de plaine et de vallées : Dufaure (C4 sommet : $10910 \pm 220$ B.P.) (Altuna et Mariezkurrena, 1995) et Duruthy (C3: $11300 \pm 1130$ B.P.), (Delpech, 1978). Ils témoignent qu'à cette époque le Renne est encore présent, à basse altitude dans les Landes, avant de se retirer, certes, mais probablement en latitude. Cette hypothèse a déjà été émise au sujet des rennes du Massif Central qui semblent avoir déserté cette région dès l'Azilien et sans refuge altitudinal temporaire (Fontana, 1996 et 1998b).

\section{CONCLUSION}

Au terme de cette étude, plusieurs éléments nouveaux sont apparus, relatifs à l'économie de chasse et aux modalités d'occupation du Bassin de l'Aude par les Magdaléniens.

La place du Renne dans l'économie alimentaire des Magdaléniens du nord de l'Aude durant environ 4000 ans (entre $16750 \pm 250$ B.P. et $12550 \pm 210$ B.P.), a peu d'équivalent en France. Une seule autre région montre cette récurrence du Renne au Badegoulien et au Magdalénien moyen, celle du Lot et de la Dordogne (Delpech, 1983 ; White, 1985 et 1989). L'exploitation du Lièvre variable à Gazel est un fait encore plus singulier dans la mesure où ce statut de gibier de prédilection n'est pas documenté sur d'autres sites magdaléniens. L'économie de chasse et probablement le type de mobilité des Magdaléniens de l'Aude semblent avoir 
été différents selon la région occupée et la période. Les sites du nord de l'Aude témoignent d'occupations hivernales probablement résidentielles pour une grande part, et d'une chasse orientée vers les troupeaux de rennes (et les lièvres variables à Gazel) regroupés à cette saison, et ceci quelle que soit la période, entre 17000 et 12300 B.P. Cette situation s'explique peutêtre par la position géographique de la Montagne Noire : elle borde une plaine qui est un passage naturel prolongeant la bordure pyrénéenne et se poursuivant vers la côte méditerranéenne. Elle est donc le passage obligé des hommes et des objets qu'ils transportent entre l'Atlantique et la Méditerranée. La variété des biotopes (plaines, collines, bord de mer), la présence de gites à silex et probablement l'abondante population de rennes, entre la fin de l'automne et le début du printemps au moins, sont autant de points qui ont pu attirer les Magdaléniens dans cette région. Il est possible que les Magdaléniens qui occupaient le Bassin de l'Aude entre 17 et 14000 B.P., en saison froide (fin de l'automne, hiver et début du printemps), occupaient une partie du Bassin de l'Ariège à la belle saison, ces deux régions étant distantes d'une centaine de kilomètres à vol d'oiseau. Néanmoins, la question de l'organisation des acquisitions et de l'étendue du territoire exploité sera plus claire lorsque l'origine des différents silex blonds sera précisée.

Dans le sud de l'Aude, les données sont bien différentes. Les sites plus modestes et plus nombreux sont uniquement postérieurs à 12500 B.P. et les chasses semblent plus diversifiées, de belle saison, avec une préférence accordée au Bouquetin. L'hiver n'est pas représenté pour l'instant dans cette région. Enfin, il est beaucoup plus délicat de savoir si une complémentarité saisonnière existait à cette période, cette fois entre les sites du nord et ceux du sud ou entre ceux du sud et les sites ariégeois (Haute Ariège), en partie en raison de le la méconnaissance de l'origine du silex et du manque de datations radiocarbones.

\section{RÉFÉRENCES BIBLIOGRAPHIQUES}

ALTUNA J. et MARIEZKURRENA K. (1995) - Les restes osseux de macromammifères, in: Straus dir., Les derniers chasseurs du monde pyrénéen. L'abri Dufaure: un gisement tardiglaciaire en Gascogne, Mém. de la Société Préhistorique Française, t. 22, p. 181-211, 8 fig., 24 tabl.

ALTUNA J. el MARIEZKURRENA K. (1996) - Faunes de mammifères des gisements magdaléniens du pays Basque et zones limitrophes, in: Pyrénées préhistoriques; Arts et sociétés, Paris, C.T.H.S., p. 149-162, 6 fig., 5 tabl.

BAHN P.G. (1983) - Pyrenean Prehistory - a palaeoeconomic survey of french sites, Warminster, Aris \& Phillips, $511 \mathrm{p}$

BEGOUEN R et CLOTTES J (1981) - Nouvelles fouilles dans la salle des Morts de la caverne d'Enlène à Montesquieu-Avantès (Ariège), in: XXI" Congrès Préhistorique de France (1979).

BARONE R. (1984) - Anatomie comparée des mammifères domestiques, t. 3: splanchnologie 1, appareil digestif et respiratoire, Paris, Vigot.

BINFORD L.R. (1978) - Nunamiut Archaeology, New-York Academic Press, 509 p.

BOUCHUD J. (1966) - Essai sur le renne et la climatologie du Paléolithique moyen et supérieur. Imprimerie R. et M. Magne, Périgueux, 300 p., 71 tabl., 55 fig., 13 pl.

BUBENIK (1989) - Teeth in the Elk of North America, Londres, Thomas \& Toweill.

CAUGHLEY G. (1966) - Mortality patterns in mammals, Ecology, t. 47 , fasc. 6 , p. 906-918, 7 fig., 2 tabl.

CLOTTES J. (1989) - Le magdalénien des Pyrénées, in : Le Magdalénien en Europe: la structuration du Magdalénien, E.R.A.U.L, t. 38 , p. $281-360$

COUTURIER A.-J. (1938) - Le chamois (Rupicapra rupicapra L.) Grenoble, B. Arthaud éd., $855 \mathrm{p}$.

COUTURIER A.-J. (1962) - Le bouquetin des Alpes, Grenoble, Imprimerie Allier éd., 1564 p., 144 pl., 21 cartes, 51 fig.

CREMADES, M. (1994) - Sédentarité et migrations animales à travers les figurations d'oiseaux de l'art paléolithque français, Préhistoire ariégeoise, t. XLIX, p. 191-212, 13 fig., 5 tabl.
DELPECH F. (1978) - Les faunes magdalénienne et azilienne du gisement de Duruthy, in: R. Arambourou éd., Le gisement préhistorique de Duruthy, Mém. la Société Préhistorique Francaise, t. 13 , p. $100-116$.

DELPECH F. (1983) - Les faunes du Paléolithique supérieur dans le Sud-Ouest de la France, C.N.R.S., Paris, Cahiers du Quaternaire, t. 6, 453 p., 71 fig., 147 tabl., 6 pl. h.t.

DELPECH F. et LE GALL O. (1983) - La faune magdalénienne de la grotte des Églises (Ussat, Ariège), Bulletin de la Société Préhistorique de l'Ariège, t. 38, p. 91-118,6 fig. 11 tabl.

DELPECH F. et VILLA P. (1993) - Activités de chasse et de boucherie dans la Grotte des Églises, in: L'exploitation des animaux sauvages à travers le temps, Antibes, A.P.D.C.A. p. 79-102, 18 fig.

DESSE-BERSET N. (inédit) - Les poissons de la grotte Gazel. Rapport inédit.

FONTANA L. (1995) - Chasseurs magdaléniens et rennes en Bassin de l'Aude: analyse préliminaire, in: L'animal dans l'espace humain. L'homme dans l'espace animal, Anthropozoologica, t. 21, p. 147-156, 5 fig., 1 tabl.

FONTANA L. (1996) - État de la recherche et problématiques en archéozoologie pour le Paléolithique supérieur d'Auvergne, in : Paléolithique supérieur entre Seine et Rhin, Cahiers Archéologiques de Bourgogne, t. 6, p. 152-160, 1 fig., 2 tabl.

FONTANA L. (1998a) - Mobilité et subsistance au Magdalénien dans le Languedoc occidental et le Roussilion. Thèse de III $^{e}$ cycle. Université de Paris I, 287 p. dact., 2 t.

FONTANA L. (1998b) - Mobilité et subsistance au Magdalénien supérieur en Auvergne, in: Économie préhistorique: les comportements de subsistance au paléolithique, Antibes, A.P.D.C.A., p. 373-386, 2 fig., 2 tabl.

FONTANA L. (1998c) - Subsistance et territoire au Magdalénien supérieur dans les Pyrénées : l'apport des données archéozoologiques de la grotte de Belvis (Aude), Préhistoire du Sud-Ouest. t. 5, fasc. 2, p. 131-146, 6 fig. 2 tabl.

FRISON G. (1970) - The Glenrock Buffalo Jump, 48C0304, Plains Anthropologist, Memoir 7 
GEDDES D., CARRERE I. et ROBERT M. (1985) - La Balma Margineda, animaux sauvages et animaux domestiques, Les Dossiers de l'Archéologie, t. 96, p. 28-30.

GEDDES D., BARBAZA M., VAQUER J. et GUILAINE J. (1986) Tardiglacial and postglacial in the eastern Pyrénées and western Languedoc (France), in: Straus dir., The end of the Paleolithic in the Old World, B.A.R. Int. Ser. t. 284, p. 63-80, 1 fig.

LALANDE B. (1986) - Contribution à l'étude des faunes magdaléniennes de la grotte d'Enlène (Ariège). Les grands mammifères de la Salle du Fond, fouilles anciennes, Diplôme d'études supérieures, Institut du Quaternaire, Université de Bordeaux I.

LE GALL O. (1992) - Les Magdaléniens et l'ichtyofaune dulçaquicole, in: Le peuplement magdalénien. Paléogéographie physique et humaine, Paris, C.T.H.S., p. 277-285, 3 fig.

LE GALL O. (1999) - Élements de réflexion sur la pêche dans le Bassin méditerranéen Nord-occidental pendant le développement des faciès leptolithiques, in: Les civilisations méditerranéennes: Les faciès leptolithiques du Bassin méditerranéen Nordoccidental : milieux naturels et culturels, Actes du XXIV Congrès préhistorique de France (Carcassonne, sept. 1994).

LEVINE M.A. (1983) - Mortality models and the interpretation of horse population structure, in: Bailey dir., Hunter-Gatherer Economy in Prehistory, Cambridge, Cambridge Univ. Press, p. $23-46,30$ fig., 5 tabl

LOWE V. (1967) - Teeth as indicators of age with special reference to Red deer Cervus Elaphus, Journ. of Zoology (Londres), t. 152, p. 137-153.

LYMAN R.L. (1987) - On the analysis of vertebrate mortality profiles: sample size, mortality type, and hunting pressure, American Antiquity, t. 52, fasc. 1, p. $125-142,8$ fig. 3 tabl.

MILLER FL (1974) - Biology of the Kaminuriak population of barrenground caribou, Part 2, Ottawa, Canadian Wildlife Service Reports, t. 36, 74 p.

MITCHELL B. (1963) - Determination of age in Scottish Red deer from growth layers in dental cement, Nature, t. 198 , p. $350-351$.

MOIGNE A.-M., BAILLS H, et GREGOIRE S. (1998) - Les Magdaléniens de la grotte de Las Conques: caractérisation du site d'après l'outillage et les restes osseux, in: Economie préhistorique: les comportements de subsistance au Paléolithique, Antibes (1997), A.D.P.C.A., p. 397-412, 8 fig., 2 tabl.

PAILHAUGUE N. (1996) - Faune et saisons de chasse de la salle Monique, grotte de la Vache (Alliat, Ariège), in: Pyrénées préhistoriques; Arts et sociétés, Paris, C.T.H.S., p. 173-191, 10 fig. 7 tabl.

PATOU M. (1984) - La faune de la galerie Rive Droite du Mas d'Azil (Ariège): données paléoclimatiques et palethnographiques, Bulletin de la Société Préhistorique Française, t. 81, p. 311-319 8 fig., 9 tabl.

PATOU M. (1985) - Dénombrement et détermination de l'âge des Rennes (Rangifer tarandus) provenant de la galerie Rive droite du Mas d'Azil (Ariège) : méthode et résultats, Revue de Paléobiologie, t. 4 , p. $71-78,4$ fig., 6 tabl.
PARKER G.R. (1972) - Biology of the Kaminuriak population of Barren-ground caribou, Part 1: Total numbers, mortality, recruitment and seasonal distribution, Ottawa, Canadian Wildlife Service, Report Series t. 20, 95 p.

PEKELHARING C.J. (1970) - Note on determining the age of the chamois (Rupicapra rupicapra L.), N. Z. For Research Institute For Prods reports, t. 73 , p. 1-6.

PEREZ-BARBERIA F. (1994) - Determination of age in Cantabrian chamois (Rupicapra pyrenaïca parva) from jaw tooth-row eruption and wear, Journ. of Zoology (Londres) t. 233, p. 649-656, 4 fig., 1 tabl.

PFLIEGER R. (1982) - Le chamois : son identification et sa vie, Paris, Gerfaut.

SACCHI D. (1986) - Le Paléolithique supérieur du Languedoc occidental et du Roussillon, $\mathrm{XXI}^{\mathrm{e}}$ suppl. à Gallia Préhistoire, Paris, C.N.R.S., 284 p., 204 fig., 16 pl. h.t., 36 tabl., 1 dépl.

SACCHI D. (1994) - Un site paléolithique supérieur de moyenne altitude dans les Pyrénées : La Cauna de Belvis (France), in: Human adaptations to the Mountain Environment in the Upper Palaeolithic and Mesolithic, Prehistoria Alpina, t. 28, fasc. 2, p. 59-89, 15 fig. 3 tabl.

STINER M.C. (1990) - The Use of Mortality Patterns in Archaeological Studies of Hominid Predatory Adaptations, Journ. of Anthropological Archaeology, t. 9, p. 305-351, 11 fig., 5 tabl.

TAVOSO A. (1987) - Le remplissage de la grotte Tournal à BizeMinervois (Aude), Cypsela, t. VI, p. 23-35, $8 \mathrm{fig}$.

VILETTE P. (1983) - Avifaunes du Pleistocène final et de l'Holocène dans le sud de la France et en Catalogne, Atacina, t. 11, 190 p. 40 fig., 3 pl. h.t., 15 cartes, 62 tabl.

WEGRZYN M., SERWATKAS S. (1984) - Teeth eruption in the Europeana Bison, Acta theriologica, t. 29, fasc. 9, p. 111-121.

WHITE R. (1985) - Upper paleolithic land use in the Périgord: a topographic approach to subsistence and settlement, Oxford, British Archaeological Report Int. Ser. 253.

WHITE R. (1989) - Husbandry and herd control in the Upper Paleolithic, a critical review of the evidence, Current Anthropology, t. 30 , 3 fig., 1 tabl., p. 609-632.

WILSON B. (1980) - Population dynamics of the Garnsey site bison, in: Late prehistoric Bison procurement in Southeastern New Mexico (Speth et Parry ed.). Ann Arbor: Museum of Anthropology, University of Michigan, Techn. Report, t. 12, p. 88-129.

Laure FONTANA

U.R.A. 1415 - C.N.R.S.

Laboratoire d'Anatomie comparée 55 , rue Buffon - 75005 Paris 